\title{
Planar solutions of higher-spin theory. Part I. Free field level
}

\section{V.E. Didenko and A.V. Korybut}

I.E. Tamm Department of Theoretical Physics, Lebedev Physical Institute, Leninsky prospect 53, Moscow 119991, Russia

E-mail: didenko@lpi.ru, akoribut@gmail.com

ABSTRACT: Many black hole solutions of General Relativity are known to be linearly exact. This opens a way to study them in gauge theories that apart from gravity contain fields of higher spin $s>2$. Starting with a black brane in $\mathrm{AdS}_{4}$ we find its free field higherspin generalization that respects static and planar symmetry for all bosonic gauge fields $s \geq 0$. The solution is found for both the higher-spin curvatures and potentials in the form suitable for further non-linear analysis and satisfies the multi copy relation.

KeYwords: Black Holes, Higher Spin Gravity, Higher Spin Symmetry

ARXIV EPRINT: 2105.09021 


\section{Contents}

1 Introduction 1

$2 \mathrm{AdS}_{4}$ black brane $\quad 5$

$\begin{array}{lll}2.1 & \text { Hidden symmetry and linearization } & 7\end{array}$

2.1.1 Brane condition 9

2.1.2 Flat space-time Penrose transform 11

2.2 Brane induced flat connection 12

3 Higher-spin equations $\quad 14$

$\begin{array}{ll}3.1 & \text { Unfolded Penrose transform } \\ \end{array}$

4 Solutions $\quad \mathbf{1 8}$

4.1 Twisted-adjoint sector 18

$\begin{array}{lll}4.2 & \text { Adjoint sector } & 21\end{array}$

5 Conclusion $\quad 24$

A Explicit form of the generating parameter $\quad 25$

B Derivation of $s p(2)$ connection $\quad 26$

$\begin{array}{ll}\text { C Checking the adjoint module } & 27\end{array}$

\section{Introduction}

Remarkable properties of black holes make them a valuable arena in different branches of theoretical physics. Particularly, within the AdS/CFT correspondence [1-3] black holes play central role in description of various thermodynamical phenomena that take place in the dual CFT theory. Glimpses of deep relation between black hole entropy and its statistical realization in terms of free fields have already been available [4], even before they plunged into gauge/gravity mainstream [5]. Lots of investigation is focused on testing different aspects of the famous correspondence which relates two theories in the opposite regimes. Given one of the two sides is strongly coupled, no wonder the duality still remains a conjecture.

Among many dual pairs there are some that relate simplest CFT vector models of $N$ free fields on the three dimensional boundary of $\mathrm{AdS}_{4}$ to a highly non-trivial higher-spin (HS) theory in the bulk [6-8]. Along with graviton the latter contains interacting scalar and gauge fields of all integer spins. As the dual CFT is supposed to be free such a duality is of weak-weak type and therefore is testable at least in perturbations. Currently this endeavor is mostly confined to tree level since the conventional HS action principle is still lacking. 
Before the AdS/CFT ages, HS theory had its own pace with some notable milestones which include the free Fronsdal Lagrangian [9], the first instance of cubic vertices found using the light-cone approach [10], the observation of relevance of AdS background for HS interactions [11], the full list of cubic interactions classified by Metsaev [12], and eventually the generating Vasiliev system for any order HS equations [13] (see [14-17] for reviews). Higher-order off-shell formulation that would make quantum analysis accessible is still not there though there are steps in this direction (see e.g., [18-21]).

It took a while since the original HS/CFT proposal was made before some checks carried out at the level of three point functions. In a remarkable feat of technical analysis Giombi and Yin [22] were able to extract certain 3pt correlators from Vasiliev equations of motion and found perfect match with the boundary expectations. With this work HS theory gained another boost that largely clarified the status of the duality (for incomplete list of references see [19, 23-32]).

Little is known however what happens to HS bulk theory in $d+1 \geq 3$ at non-zero temperature $T>0$. Typically of gravity theories, the CFT dual thermal states with $T<T_{c}$ are associated with planar black holes that radiate Hawking temperature $T_{\mathrm{H}}$. It originates from a factorization that removes a deficit angle in the Euclidian version of a black hole metric thus introducing a thermal $S^{1}$. For the boundary theory on a sphere a large spherical black hole in the bulk corresponds to a thermal state beyond a critical point $T>T_{c}$, where a phase transition occurs.

HS theory is very much different as its spin two sector and the corresponding metric seems to have no invariant meaning under higher-spin symmetry transformation. This makes the very notion of horizon debatable. Even in the case of $\mathrm{HS}_{3} / \mathrm{CFT}_{2}$ duality [33-35] which is much more elaborated due to its topological nature and the fact that the HS field spectrum can be made finite, the role of $\mathrm{HS}_{3}$ black hole horizon [36] (if any) is unclear.

Besides, as Shenker and Yin pointed out in [37], for $d=3 \mathrm{U}(N)$ vector model on $S^{2} \times S^{1}$ the phase transition takes place at Plankian temperature $T \sim \sqrt{N}$ rather than at AdS scale $T \sim 1$ in sharp contrast with a field theory in the adjoint. This fact seemingly indicates the absence of uncharged spherically symmetric black hole in $\mathrm{AdS}_{4}$ HS theory. This might not be too surprising after all, as generally in HS theory lower spins source higher spins and $s=2$ is not an exception. Whether it is so with a black brane or not will be investigated in [38] at the lowest interaction order. But general expectation is that a potential HS black hole may have infinitely many charges. This may result in smearing off the AdS scale phase transition due to infinitely many black hole states. All in all, the idea of associating thermal states of HS theory to its exact solutions with proper global symmetry still seems natural.

The goal of this paper is to set the stage for perturbative analysis of static HS solutions in four dimensions that in addition have planar symmetry and to find the associated solutions at free level. In other words we are interested in HS counterparts of black branes in perturbations. The problem is considered within the Vasiliev theory.

One of the reasons for perturbative analysis rather than the all order one is the HS locality problem that for the time being has not been yet solved to all orders. To be more specific it is not clear whether HS vertices for a given set of spins contain finite number 
of derivatives beyond cubic order or not [39, 40]. This problem is a daunting challenge within the holographic approach that allows one at least in principle reconstructing bulk interactions from the free boundary CFT correlators. This idea was proposed by Petkou in [41] as he anticipated that the procedure of reconstructing quartic scalar interaction would be straightforward if perhaps technical. It was solved later on in [39]. At quartic level one encounters non-localities that somehow drives the analysis out of control [40]. Indeed, as was pointed out in [40, 42], admitting wild non-localities in field redefinition trivializes the Noether procedure and formally reduces interacting theory to the free one.

The state of affairs with $d+1 \geq 4$ Vasiliev theory is less uncertain although the (non)locality problem is a pressing issue too. The great advantage of the Vasiliev approach is that the HS equations it reproduces are given in any HS background rather than on AdS. On a practical note this renders the perturbation theory pretty peculiar being exact in HS 1-form potentials $\omega$ while decomposable in terms of HS Weyl tensors $C$. It turns out in particular that as shown in [43] a cubic vertex (quadratic in $C$ ) can not be zero once the linear one in $C$ is present. This implies that there are no field redefinitions that allows one getting rid of cubic vertex for if there were, the resulting system would be either formally inconsistent or contained no free equations. The latter option can not be realized for cohomological reason. This seemingly leaves no room for any ambiguity in calculation of boundary observables from the bulk. Once they are finite they can be checked against CFT expectations. The non-admissible non-locality results into infinities for these quantities. An urgent problem is therefore to understand a class of admissible field variables that render obserbvables finite.

Being formulated in a certain twistor space a natural substitute for the space-time locality in this approach is a twistor locality proposed in [44] and coined the spin-locality which if present guarantees finiteness of classical theory. In a series of papers [44-50] the spin-locality conjecture was confirmed ${ }^{1}$ for many non-linear Vasiliev vertices but still those do not yet cover the full quartic vertex on AdS. It is for this reason that we confined to perturbative analysis in our quest for planar solutions.

Despite the locality issue, there is a handful of exact solutions for Vasiliev equations in four dimensions that were constructed over the years [51-59]. Particularly, in [53] a static solution with spherical symmetry was found. In many respects it can be seen as a generalization of an extremal black hole. Having infinitely many HS parameters all equal to each other it generalizes the mass equals charge relation for usual extremal black hole. At free level the $s=2$ Weyl tensor appears to be the one of the Schwarzschild solution. The solution preserves some amount of supersymmetries. In [60] it was considered as embeddings in different susy HS models. In [54] the extremality condition was relaxed such that HS parameters entering the solution become arbitrary thus presenting a new family of black hole like solutions in HS theory (see [58] for review).

While it seems not unlikely that the form of some of the obtained exact solutions should be reconsidered in view of the locality issue, some interesting information on their properties

\footnotetext{
${ }^{1}$ The observed structure of spin-locality turned out to have a certain $Z_{2}$-graded form. While spin-local structures can generate non-local ones in star-product commutators, in practice they do not for among two of them within a star-product commutator at least one appears to be spin ultra-local [46] yielding no non-localities.
} 
can still be gained at free level. Particularly, a remarkable boundary interpretation of the linearized version of HS extremal black hole as a bi-local operator on the boundary was established in [61]. The same authors have also observed that a BPS-like pattern of the solution results in certain UV-cancellations of two interacting such black hole states at leading order.

A powerful method for generating HS analogs of black hole like solutions at linear level rests on the observation from [62]. It was shown there that a vast class of Petrov $D$-type solutions of General Relativity in four dimensions that include Kerr, planar, etc. black holes are generated with a single AdS global symmetry parameter. ${ }^{2}$ These naturally generalize the double copy form to a multi copy one. From a twistor standpoint this statement is equivalent to having some constant rank-2 dual twistor that generates solutions to free massless equations via the Penrose transform [53] (see also [64, 65]). The resulting Weyl tensors are of the generalized $D$-type. For the pure gravity case a linearized Riemann tensor obtained this way is in fact exact. The origin of this linearization is a hidden symmetry of $D$-type metrics as they admit the so called Killing-Yano tensor [66, 67]. This property allows one naturally incorporate the $s=2$ black hole solutions into the linearized HS theory. The unfolded version of the Penrose transform well adopted for HS analysis has been introduced in [53,68]. It plays an important role in HS solution generating techniques $[53,54,56,59]$ as well as in HS/CFT analysis [31, 69].

The main results of our paper are the following. Using the proper $\mathrm{AdS}_{4}$ global symmetry parameter ${ }^{3}$ (rank-2 dual twistor) we generate solutions for the free bosonic HS fields $s \geq 0$ on the AdS background via the unfolded Penrose transform. These solve the so called 0-form sector (Weyl module) of the linearized HS equations. We then restore the HS potentials corresponding to the 1-form sector of HS equations for the case of HS parity even model $(\eta=\bar{\eta}=1)$. The obtained solutions are of $D$-type, static and admit spatial planar symmetry similar to that of a black brane. Moreover, the spin $s=2$ sector is exactly equal to a black brane Weyl tensor in agreement with the well known result from gravity.

While solving equations for the Weyl module $C$ using the Penrose-like trick is not a problem, to recover the corresponding HS potential sector $\omega$ which is sourced by $C$ in a form suitable for higher orders is not always an easy job. The problem is somewhat equivalent to recover the metric from its Riemann tensor. The procedure is gauge dependent. Even at free level the result can be quite complicated compared to the form of the original source $C$ (see e.g., [71, 72]). For the planar solutions of our primary interest we find surprisingly simple result for fields $\omega$ in the parity even HS model with $\eta=1$. On a technical side, one reason why a simplification takes place is the existence of an auxiliary $s p(2)$ flat connection induced by the generating symmetry parameter (dual twistor) that results in a natural ansatz for $\omega$ which otherwise might be difficult to grasp. The use of such a flat connection is beneficial at higher orders as well [38] and plays a key role in our analysis.

Our solution is characterized by infinitely many parameters attributed to different individual fields of given spin and parameterized by an arbitrary analytic in the generating

\footnotetext{
${ }^{2}$ In fact the most general $D$-type Plebanski-Demianski metric can be described this way by making the AdS symmetry parameter not hermitian conjugate [63].

${ }^{3}$ See [70] for incomplete classification of such parameters.
} 
spinor variables function. This function is polynomial in the case of a finite amount of massless fields and non-polynomial otherwise, which is a feature of the linearized approximation. An interesting property of the obtained solution is the unique star-product Fock projector that shows up as a factor for every spin $s$ field within the Weyl module. Generally, Fock projectors play crucial role in HS bulk-boundary analysis (see $[22,30,73,74]$ ). Particularly, HS boundary-to-bulk propagators are of this form [75]. They as well play a role of a probe data for possible non-localities within the HS equations producing infinities for non-local self-interaction. ${ }^{4}$ At the nonlinear level Fock projectors tend to factorize (twisted)-adjoint HS equations into left and right modules. It would be very interesting to carry out higher order analysis of such solutions especially within the context of bulkboundary analysis of [30], where a Fock projector naturally appears. At quadratic level the HS corrections to the planar solution will be analyzed in [38].

The layout of the paper is as follows. In section 2 we give a detailed description of algebraic properties of $\mathrm{AdS}_{4}$ black brane. We show how its linearized nature results from hidden symmetry attributed to a Killing-Yano tensor. It exists in the vacuum background whether it is Minkowski or AdS and along with the Killing symmetry forms a global symmetry parameter that builds up geometry of a black hole. We then give a condition on that parameter to correspond to the planar symmetry. Then we briefly review the Penrose transform that treats this parameter as a rank-two twistor and allows one generating solutions for any spin $s \geq 0$. We conclude this section with the description of the brane induced $s p(2)$ flat connection that will play a distinguished role in our analysis of the solutions of HS equations. In section 3 the linearized Vasiliev HS equations are reviewed and the unfolded version of the Penrose transform is introduced. In section 4 we find solutions of these equations and we conclude in section 5. The paper is supplemented with three appendices that contain the explicit form of the generating global symmetry parameter in the Poincare chart, the derivation of the $s p(2)$ connection and, finally, the details on derivation of our solution in the sector of HS potentials.

\section{$2 \quad \mathrm{AdS}_{4}$ black brane}

The presence of negative cosmological constant affects drastically classical topology theorems resulting in that black holes in asymptotically AdS space may have different horizon topologies. Apart from the usual positive curvature horizon that Kerr solution has there can be horizons of negative and zero curvatures. These are the hyperbolic and planar black holes respectively. In fact, in four dimensions an arbitrary genus Riemann surface horizon is possible as one can quotient the hyperbolic horizon over a discrete subgroup. We will be interested in a planar black hole here the metric of which can be chosen in the following standard form

$$
d s^{2}=-\frac{d r^{2}}{\Lambda r^{2}+M / r}+\left(\Lambda r^{2}+M / r\right) d t^{2}-\Lambda r^{2}\left(d x^{2}+d y^{2}\right)
$$

\footnotetext{
${ }^{4}$ Particularly, early analysis of HS black brane at non-linear level has revealed some pathologies within the non-local setup [76].
} 
where $\Lambda$ is the cosmological constant. When

$$
\Lambda<0
$$

there is a horizon at

$$
r_{0}=\left(-\frac{M}{\Lambda}\right)^{\frac{1}{3}}
$$

where $M>0$ is a massive parameter of the AdS black brane. Metric (2.1) is manifestly time independent which implies that the solution is stationary and in fact static. It as well has no dependence on $x$ and $y$ meaning that there is another set of isometries that leave the two dimensional spatial plane

$$
d l^{2}=d x^{2}+d y^{2}
$$

invariant. These form the $i s o(2)$ algebra and along with time translation generate $u(1) \oplus$ $i s o(2)$ isometry algebra that can be realized using vector fields

$$
\begin{array}{rlrl}
u(1): & T & =\frac{\partial}{\partial t}, & \\
\text { iso(2) }: & P_{1}=\frac{\partial}{\partial x}, & P_{2}=\frac{\partial}{\partial y}, \quad L=x \frac{\partial}{\partial y}-y \frac{\partial}{\partial x}
\end{array}
$$

with the commutation relations

$$
\left[T, P_{1,2}\right]=[T, L]=0, \quad\left[P_{1}, P_{2}\right]=0, \quad\left[P_{1}, L\right]=P_{2}, \quad\left[P_{2}, L\right]=-P_{1}
$$

When $M=0,(2.1)$ reduces to the $\mathrm{AdS}_{4}$ Poincare chart

$$
d s^{2}=\frac{1}{z^{2}}\left(-d t^{2}+d x^{2}+d y^{2}+d z^{2}\right), \quad z=\frac{1}{\sqrt{-\Lambda} r} .
$$

In these coordinates the spatial planar symmetry (2.6) can be realized as the centralizer of $T=\frac{\partial}{\partial t} \in \operatorname{so}(3,2)$ which fact will be useful in what follows.

Four dimensional black holes in general and solution (2.1) in particular share the linearization property. Namely, being exact solutions to non-linear Einstein equations they at the same time satisfy the linearized ones. Moreover, the non-linear corrections are satisfied as a consequence of the free solutions. This property can be envisaged from the form of a black hole Weyl tensor which is linear in $M$. The statement can be made precise by means of the Kerr-Schild ansatz

$$
g_{m n}=g_{0 m n}+\frac{M}{U} l_{m} l_{n},
$$

which decomposes black hole metric $g_{m n}$ in a sum of the AdS background $g_{0 m n}$ and the linear in $M$ fluctuation part made of shear free geodesic congruence $l^{m}$

$$
l_{m} l^{m}=0, \quad l^{m} D_{m} l_{n}=0
$$

and some scalar function $U$. Index contraction in (2.10) is carried out with respect to either background or full metric. The covariant derivative $D_{m}$ can be also attributed to either 
metric. Equations (2.10) as well as the linearized Einstein equations for (2.9) guarantee that linear in $M$ approximation is exact. This behavior can be understood as follows. Looking at black hole metric as some deformation of the AdS space, the linearization property suggests that certain background geometry characteristics remain undeformed implying that the black hole curvature is made of the AdS background remnants. This remnant appears to be a Killing-Yano tensor (see [77] for a comprehensive review) that $D$-type metrics have which is present in AdS (and flat) space-time and remains unchanged upon deformation. Since the AdS background Killing-Yano does not depend on $M$ the black hole Riemann tensor it provides has no any dependence on $M$ other than through a linear in $M$ overall factor. The existence of the Killing-Yano tensor in black hole geometry is often referred to as a hidden symmetry, while its description is most accessible in the language of two-component spinors.

\subsection{Hidden symmetry and linearization}

A convenient way to describe black holes that well captures their algebraic properties is by using Cartan formalism. Consider Cartan structure equations

$$
\begin{aligned}
\mathrm{d} \mathbf{w}_{\mathbf{a b}}+\mathbf{w}_{a}{ }^{c} \mathbf{w}_{c b} & =\mathbf{R}_{\mathbf{a b}}, \\
D \mathbf{e}_{a} \equiv \mathrm{de}_{a}+\mathbf{w}_{a}{ }^{b} \mathbf{e}_{b} & =0 .
\end{aligned}
$$

Here fields $\mathbf{w}_{a b}=-\mathbf{w}_{b a}$ and $\mathbf{e}_{a}$ are the one-forms of Lorentz connection and vierbein respectively. The two-form $\mathbf{R}_{a b}$ is the Riemann curvature. Indices are contracted using the Minkowski metric $\eta_{a b}$.

The notion of hidden symmetry in a certain sense is a natural generalization of Killing symmetries. Suppose one has a Lorentz vector $t^{a}$. The action of covariant differential (2.12) on it generally results in

$$
D t_{a}=\mathbf{e}^{b} s_{a b}+\mathbf{e}^{b} n_{a b},
$$

where $s_{a b}=s_{b a}$ and $n_{a b}=-n_{b a}$ are some (anti)symmetric tensors that together form the most general right hand side of (2.13). If one of those tensors is absent then the space-time may have a (hidden) symmetry. For example, if $s_{a b}=0$, then $t^{a}$ is a Killing vector. Indeed, since $n_{a b}$ is antisymmetric, equation (2.13) is equivalent to

$$
D_{a} t_{b}+D_{b} t_{a}=0
$$

which is just the Killing equation. If only the traceless part of $s_{a b}$ is missing in (2.13), then the resulting condition would imply $t^{a}$ to be a conformal Killing vector. If instead $n_{a b}=0$, then (2.13) is equivalent to $\partial_{[a} t_{b]}=0$ which sets no restriction on geometry.

Similarly, one can consider more complicated tensor structures in place of $t^{a}$, such as an antisymmetric tensor $t_{a b}=-t_{b a}$ for which one can write down

$$
D t_{a b}=\mathbf{e}^{c} r_{a b c}+\mathbf{e}^{c} h_{a b, c}+\mathbf{e}_{[a} n_{b]},
$$


where the right hand side of $(2.15)$ is decomposed in terms of irreducible so(3,1) traceless Young diagrams ${ }^{5}$

$$
\begin{aligned}
r_{a b c} & =r_{[a b c]}, \\
h_{a b, c} & =h_{[a b], c}, \quad h_{[a b, c]}=0, \quad \eta^{a c} h_{a b, c}=0 .
\end{aligned}
$$

$t_{a b}$ is said to be a rank-2 Killing-Yano tensor (KY) if $h_{a b, c}=0$ and $n_{a}=0$ being a natural generalization for Killing condition (2.14). Analogously, if only $h_{a b, c}=0$ then such $t_{a b}$ is a conformal KY. If instead $r_{a b c}=0$ and $h_{a b, c}=0$, then $t_{a b}$ is called a closed conformal KY. A higher rank totally antisymmetric tensor corresponds to a higher-rank KY. There are no such structures in four dimensions though.

The integrability requirement for (2.11)-(2.12) $D^{2} \sim \mathbf{R}_{\mathbf{a b}}, D \mathbf{R}_{a b}=0$ severely constrains any symmetry, so that a generic space-time has no KY's. The simplest example that admits KY symmetry is AdS (or flat) space-time, where it has a straightforward interpretation. To this end consider system (2.11)-(2.12) for AdS background

$$
\begin{aligned}
\mathrm{d} \mathbf{w}_{\mathbf{a b}}+\mathbf{w}_{a}{ }^{c} \mathbf{w}_{c b} & =\Lambda \mathbf{e}_{a} \mathbf{e}_{\mathbf{b}}, \\
\mathrm{d} \mathbf{e}_{a}+\mathbf{w}_{a}{ }^{b} \mathbf{e}_{b} & =0 .
\end{aligned}
$$

A nice feature of this system is that it has local gauge symmetry

$$
\begin{aligned}
\delta \mathbf{w}_{a b} & =D \varkappa_{a b}+\Lambda\left(v_{a} \mathbf{e}_{b}-v_{b} \mathbf{e}_{a}\right), \\
\delta \mathbf{e}_{a} & =D v_{a}-\varkappa_{a b} \mathbf{e}^{b},
\end{aligned}
$$

where $\varkappa_{a b}=-\varkappa_{b a}$ and $v_{a}$ are arbitrary space-time dependent parameters. By setting $\delta \mathbf{w}_{a b}=0$ and $\delta \mathbf{e}_{a}=0$ one fixes the AdS global symmetry

$$
\begin{aligned}
D v_{a} & =\mathbf{e}^{b} \varkappa_{a b}, \\
D \varkappa_{a b} & =-\Lambda\left(v_{a} \mathbf{e}_{b}-v_{b} \mathbf{e}_{a}\right) .
\end{aligned}
$$

Here one identifies $v_{a}$ with a Killing vector, while $\varkappa_{a b}$ with a closed conformal KY. In AdS the two fields go hand in hand as parts of a global symmetry parameter, which can be naturally written as an $s o(3,2)$ covariantly constant matrix $K_{I J}=-K_{J I}$

$$
D_{0} K_{I J}=0, \quad K_{I J}=\left(\begin{array}{cc}
\varkappa_{a b} & \sqrt{-\Lambda} v_{c} \\
-\sqrt{-\Lambda} v_{c} & 0
\end{array}\right)
$$

where

$$
D_{0}=d+\mathbf{w}_{I J}, \quad \mathbf{w}_{I J}=\left(\begin{array}{cc}
\mathbf{w}_{a b} & \sqrt{-\Lambda} \mathbf{e}_{c} \\
-\sqrt{-\Lambda} \mathbf{e}_{c} & 0
\end{array}\right) .
$$

For metric (2.8) with $x^{\mu}=(t, x, y, z)$ choosing vierbein $e_{a, \mu}=\frac{1}{z} \eta_{a \mu}$ and taking Killing vector $(2.5) v^{\mu}=(1,0,0,0)$ and setting $\Lambda=-1$ for convenience we find from $(2.22)$

$$
\varkappa_{a b}=\frac{1}{z}\left(\begin{array}{cccc}
0 & 1 & 0 & 0 \\
-1 & 0 & 0 & 0 \\
0 & 0 & 0 & 0 \\
0 & 0 & 0 & 0
\end{array}\right), \quad v_{a}=\frac{1}{z}(1,0,0,0) .
$$

\footnotetext{
${ }^{5}$ In four dimensions $r_{a b c}$ can be dualized to a vector $v_{a}$ making the first and third terms on the r.h.s. of (2.15) equivalent. But to set the nomenclature we are working in generic dimension for a while.
} 
Black holes in $d=4$ and their $D$-type generalizations in higher dimensions admit a nonzero closed conformal KY much as the AdS background does. This means that (2.23) still applies for black hole covariant derivative $D$, while (2.22) should be modified because system (2.22)-(2.23) is consistent in AdS only. A consistent deformation of that system was studied in [62] using spinor language, where it was shown that a general global symmetry parameter $K_{I J}$ produces the Carter-Plebanski family of $D$-type metrics which includes all black hole solutions. Particularly, the Riemann tensor turns out to be built out of closed conformal KY field $\varkappa_{a b}$ whereas the massive parameter comes out as an overall factor. The fact that (2.23) stays the same for black holes explains their linearized nature, while smooth deformation of (2.22)-(2.23) results in a certain integrating flow that reconstructs black hole geometry in terms of the AdS global symmetry parameter (2.24) [62]. Different parameters correspond to different types of black holes. The one corresponding to the planar type has special algebraic properties that we specify using spinors.

\subsubsection{Brane condition}

Isomorphism $s o(3,2) \sim s p(4)$ allows us using the two-component spinor language. In these terms symmetry parameter $K_{I J}(2.24)$ is equivalent to a symmetric $s p(4)$ matrix $K_{A B}=K_{B A}, A, B=1, \ldots, 4$. Let us also set cosmological constant to a number for convenience, such that

$$
K_{A B}=\left(\begin{array}{cc}
\varkappa_{\alpha \beta} & v_{\alpha \dot{\beta}} \\
v_{\beta \dot{\alpha}} & \bar{\varkappa}_{\dot{\alpha} \dot{\beta}}
\end{array}\right), \quad \varkappa_{\alpha \beta}=\varkappa_{\beta \alpha}, \quad \bar{\varkappa}_{\dot{\alpha} \dot{\beta}}=\bar{\varkappa}_{\dot{\beta} \dot{\alpha}}
$$

whereas $(2.22)-(2.23)$ reduce to

$$
\begin{aligned}
D \varkappa_{\alpha \beta} & =\mathbf{e}_{\alpha}{ }^{\dot{\gamma}} v_{\beta \dot{\gamma}}+\mathbf{e}_{\beta}{ }^{\dot{\gamma}} v_{\alpha \dot{\gamma}}, \\
D v_{\alpha \dot{\alpha}} & =\mathbf{e}_{\alpha}{ }^{\dot{\gamma}} \bar{\varkappa}_{\dot{\alpha} \dot{\gamma}}+\mathbf{e}^{\gamma} \dot{\alpha} \varkappa_{\gamma \alpha}
\end{aligned}
$$

or in a manifestly $\mathrm{AdS}_{4}$ covariant way

$$
D_{0} K_{A B}=0
$$

where $D_{0}=\mathrm{d}+\Omega$,

$$
\Omega_{A B}=\left(\begin{array}{cc}
\omega_{\alpha \beta} & \mathbf{e}_{\alpha \dot{\beta}} \\
\mathbf{e}_{\beta \dot{\alpha}} & \bar{\omega}_{\dot{\alpha} \dot{\beta}}
\end{array}\right) .
$$

Indices $\alpha, \dot{\alpha}=1,2$ are contracted with the help of $s p(2)$ forms $\epsilon_{\alpha \beta}=-\epsilon_{\beta \alpha}$ and $\epsilon_{\dot{\alpha} \dot{\beta}}=-\epsilon_{\dot{\beta} \dot{\alpha}}$. Formally, system (2.28)-(2.29) allows one generating the $D$-type (anti)self-dual parts of the Weyl tensor as follows

$$
C_{\alpha \beta \gamma \delta}=\frac{M}{r^{5}} \varkappa_{(\alpha \beta} \varkappa_{\gamma \delta)}, \quad \bar{C}_{\dot{\alpha} \dot{\beta} \dot{\gamma} \dot{\delta}}=\frac{\bar{M}}{\bar{r}^{5}} \bar{\varkappa}_{(\dot{\alpha} \dot{\beta}} \bar{\varkappa}_{\dot{\gamma} \dot{\delta})},
$$

where

$$
r^{2}=-\frac{1}{2} \varkappa_{\alpha \beta} \varkappa^{\alpha \beta}, \quad \bar{r}^{2}=-\frac{1}{2} \bar{\varkappa}_{\dot{\alpha} \dot{\beta}} \bar{\varkappa}^{\dot{\alpha} \dot{\beta}}
$$


and $M$ is an arbitrary parameter. Equations (2.32) correspond in particular to AdS-Kerr black hole and encompasses generic $D$-case. A consistent deformation that drives (2.28)(2.29) away from AdS, $\hat{D}^{2} \neq \Lambda$ ee looks as follows

$$
\begin{aligned}
\hat{D} \varkappa_{\alpha \beta} & =\mathbf{e}_{\alpha}{ }^{\dot{\gamma}} v_{\beta \dot{\gamma}}+\mathbf{e}_{\beta}{ }^{\dot{\gamma}} v_{\alpha \dot{\gamma}}, \\
\hat{D} v_{\alpha \dot{\alpha}} & =\bar{\rho} \mathbf{e}_{\alpha} \dot{\gamma} \bar{\varkappa}_{\dot{\alpha} \dot{\gamma}}+\rho \mathbf{e}^{\gamma} \dot{\alpha} \varkappa_{\gamma \alpha},
\end{aligned}
$$

where $\rho$ and $\bar{\rho}$ are certain functions that depend on deformation parameters (mass, NUT and electro-magnetic charges) and on $r$ and $\bar{r}$ from (2.33) (see [62]). Note that (2.34) remains unchanged thus preserving $\varkappa_{\alpha \beta}$ as a KY symmetry and providing Weyl tensors (2.32) to be expressed in terms of background fields.

While (2.32) reproduces Weyl tensors for any $K_{A B}$ from (2.27), the case of a black brane corresponds (see [70]) to real $M$ and

$$
K_{A}^{C} K_{C B}=0, \quad \operatorname{det} \varkappa=\frac{1}{2} \varkappa_{\alpha \beta} \varkappa^{\alpha \beta}<0
$$

with the following reality conditions imposed

$$
K_{A B}^{\dagger}=K_{A B}, \quad \varkappa_{\alpha \beta}^{\dagger}=\bar{\varkappa}_{\dot{\alpha} \dot{\beta}}, \quad v_{\alpha \dot{\beta}}^{\dagger}=v_{\beta \dot{\alpha}} .
$$

In this case (2.32) corresponds to a black brane which metric is given by (2.1). Condition (2.36) is $s p(4)$ invariant with respect to the adjoint group action and will be referred to as the brane condition. Let us also note the $s p(4)$ subalgebra $\varepsilon$ that commutes with $K_{A B}$

$$
[\varepsilon, K]=0
$$

spans planar symmetry $(2.5)$ and (2.6), where $u(1)=\frac{\partial}{\partial t}$ part is generated by $K$ itself (see appendix). Component form of (2.36) amounts to

$$
\begin{aligned}
\varkappa_{\alpha}^{\gamma} \varkappa_{\gamma \beta}+v_{\alpha}^{\dot{\gamma}} v_{\beta \dot{\gamma}} & =0, \\
\varkappa_{\alpha}^{\gamma} v_{\gamma \dot{\beta}} & =\bar{\varkappa}_{\dot{\beta}}^{\dot{\gamma}} v_{\alpha \dot{\gamma}}, \\
\bar{\varkappa}_{\dot{\alpha}}^{\dot{\gamma}} \bar{\varkappa}_{\dot{\gamma} \dot{\beta}}+v_{\dot{\alpha}}^{\gamma} v_{\gamma \dot{\beta}} & =0,
\end{aligned}
$$

which entails

$$
r^{2}=-\frac{1}{2} \varkappa_{\alpha \beta} \varkappa^{\alpha \beta}=-\frac{1}{2} \bar{\varkappa}_{\dot{\alpha} \dot{\beta}} \bar{\varkappa}^{\dot{\alpha} \dot{\beta}}=\frac{1}{2} v_{\alpha \dot{\alpha}} v^{\alpha \dot{\alpha}}>0 .
$$

From (2.28) we also find that

$$
\mathrm{d} r=\frac{1}{r} \mathbf{e}_{\alpha \dot{\alpha}} \varkappa^{\alpha \gamma} v_{\gamma}^{\dot{\alpha}}
$$

System (2.28)-(2.29) generates solutions to the free $s=2$ equations. For example, the Kerr-Schild vector that appears in (2.9) as well as function $U$ can be expressed as

$$
l_{\alpha \dot{\alpha}}=\frac{1}{r^{2}}\left(v_{\alpha \dot{\alpha}}+\frac{1}{r} \varkappa_{\alpha}{ }^{\beta} v_{\beta \dot{\alpha}}\right), \quad \frac{1}{U}=r .
$$

It generates massless $s \geq 0$ multi copy solutions too [78]. A comprehensive reason for this phenomenon rests on the fact that (2.28)-(2.29) is the rank-2 twistor equation. Therefore the Penrose transform can be applied to produce a tower of massless solutions [53]. While we are going to use the unfolded version of the Penrose transform specified to AdS in what follows, let us now demonstrate briefly following [65] how the standard Penrose transform results in $D$-type solutions in flat space-time. 


\subsubsection{Flat space-time Penrose transform}

Suppose we are in flat space $\Lambda=0$ and therefore $D^{2}=0$ and the vierbein can be chosen to be $\mathbf{e}^{\alpha \dot{\beta}}=\mathrm{d} x^{\alpha \dot{\beta}}$. A pair of spinors $Z^{A}=\left(\xi^{\alpha}, \bar{\xi}_{\dot{\alpha}}\right)$ is called a rank-1 twistor if the following condition is satisfied

$$
D \xi^{\alpha}=-i \mathbf{e}^{\alpha \dot{\alpha}} \bar{\xi}_{\dot{\alpha}}
$$

In the Cartesian reference frame it can be solved via

$$
\xi^{\alpha}=\xi_{0}^{\alpha}-i x^{\alpha \dot{\alpha}} \bar{\xi}_{0 \dot{\alpha}}, \quad \bar{\xi}_{\dot{\alpha}}=\bar{\xi}_{0 \dot{\alpha}}
$$

where $Z_{0}^{A}=\left(\xi_{0}^{\alpha}, \bar{\xi}_{0} \dot{\alpha}\right)$ is $x$-independent. The incidence relation

$$
\xi_{0}^{\alpha}=i x_{0}^{\alpha \dot{\alpha}} \bar{\xi}_{0 \dot{\alpha}}
$$

then allows one establishing non-local correspondence between points in space-time $x^{\alpha \dot{\alpha}}$ and points in twistor space $Z^{A}$. Similarly, one can define a dual twistor $Y_{A}=\left(\eta_{\alpha}, \bar{\eta}^{\dot{\alpha}}\right)$ via hermitian conjugation of (2.45). Tensor product of relations (2.45) and their conjugate results in an arbitrary rank twistor $Z^{A_{1} \ldots A_{n}} B_{1} \ldots B_{m}$. We can now define the Penrose transform as a map from holomorphic twistor functions of $Z^{A}=\left(u^{\alpha}, \bar{u}_{\dot{\alpha}}\right)$ into solutions of free massless equations for (HS) Weyl tensors

$$
\bar{C}_{\dot{\alpha}_{1} \ldots \dot{\alpha}_{2 s}}=\left.\oint_{\Gamma} \mathrm{d} \bar{u}^{\dot{\beta}} \bar{u}_{\dot{\beta}} \bar{u}_{\dot{\alpha}_{1}} \ldots \bar{u}_{\dot{\alpha}_{2 s}} f(Z)\right|_{C P^{1}},
$$

where the projection to $C P^{1}$ means that incidence relation (2.47) for twistor $Z^{A}$ is imposed. This implies that function $f(Z)$ depends on $\bar{u}$ variable only

$$
\left.f(Z)\right|_{C P^{1}}=f\left(i x^{\alpha \dot{\alpha}} \bar{u}_{\dot{\alpha}}, \bar{u}_{\dot{\beta}}\right)
$$

The contour $\Gamma$ is chosen to separate poles in such a way that the integration makes sense. It is straightforward to check now that the free massless spin $s$ equations hold

$$
\frac{\partial}{\partial x^{\alpha \dot{\beta}}} \bar{C}_{\dot{\alpha}_{2} \ldots \dot{\alpha}_{2 s}}^{\dot{2}}=0
$$

To generate $D$-type free Weyl tensors it is sufficient to take

$$
f_{s+1}=\frac{1}{\left(K_{0 A B} Z^{A} Z^{B}\right)^{1+s}}
$$

where $K_{0 A B}=K_{0 B A}$ is some constant rank-2 dual twistor. For a particular $K_{0}$ corresponding to Taub-NUT case ${ }^{6}$ integration (2.48) has been explicitly carried out in [65] with the final result being in agreement with the general analysis of [62].

\footnotetext{
${ }^{6}$ In classification of [70] this corresponds to $K_{A}{ }^{C} K_{C}{ }^{B}=\delta_{A}{ }^{B}$.
} 


\subsection{Brane induced flat connection}

Getting back to $\Lambda<0$ case, most symmetric black holes of spherical, planar or hyperbolic horizons are singled out by a specific symmetry parameter $K$ that satisfies

$$
K_{A}{ }^{C} K_{C}{ }^{B}=\epsilon \delta_{A}^{B},
$$

where $\epsilon=-1,0,1$ correspondingly [70]. Quite remarkably, in each case there exists an $s p(2)$ flat connection that originates from mixing Lorentz components of $\mathrm{AdS}_{4}$ connection $\Omega(2.31)$ and $K(2.27)$. In the planar case $\epsilon=0$ the form of such a connection is especially simple. ${ }^{7}$ One can make sure using (2.28)-(2.29) and (2.36) (for more detail see appendix) that the following connection is $s p(2)$ flat

$$
w_{\alpha \beta}=\omega_{\alpha \beta}+\frac{1}{2}\left(\mathbf{e}_{\alpha} \dot{\gamma} k_{\beta \dot{\gamma}}+\mathbf{e}_{\beta} \dot{\gamma} k_{\alpha \dot{\gamma}}\right),
$$

where

$$
k_{\alpha \dot{\alpha}}=-\frac{1}{r^{2}} \varkappa_{\alpha}{ }^{\beta} v_{\beta \dot{\alpha}}, \quad k_{\alpha}{ }^{\dot{\gamma}} k_{\beta \dot{\gamma}}=\epsilon_{\alpha \beta}, \quad k_{\dot{\alpha}}^{\gamma} k_{\gamma \dot{\beta}}=\epsilon_{\dot{\alpha} \dot{\beta}}
$$

and $\omega_{\alpha \beta}$ is the holomorphic part of the AdS Lorentz connection. Similarly, one defines the dual connection

$$
\bar{w}_{\dot{\alpha} \dot{\beta}}=\bar{\omega}_{\dot{\alpha} \dot{\beta}}+\frac{1}{2}\left(\mathbf{e}_{\dot{\alpha}}^{\gamma} k_{\gamma \dot{\beta}}+\mathbf{e}_{\dot{\beta}}^{\gamma} k_{\gamma \dot{\alpha}}\right) .
$$

This makes the following differential

$$
\nabla A_{\alpha \dot{\alpha}}=\mathrm{d} A_{\alpha \dot{\alpha}}-w_{\alpha}^{\beta} A_{\beta \dot{\alpha}}-\bar{w}_{\dot{\alpha}}^{\dot{\beta}} A_{\alpha \dot{\beta}}
$$

indeed nilpotent

$$
\nabla^{2}=0
$$

implying the $s p(2)$ flatness condition

$$
\mathrm{d} w_{\alpha \beta}-w_{\alpha}^{\gamma} w_{\beta \gamma}=0 .
$$

An advantage of this connection is that it makes the properly rescaled components of $(2.27)$ covariantly constant with respect to $\nabla$

$$
\nabla\left(\frac{\varkappa_{\alpha \beta}}{r}\right)=0, \quad \nabla\left(\frac{v_{\alpha \dot{\beta}}}{r}\right)=0, \quad \nabla\left(\frac{\bar{\varkappa}_{\dot{\alpha} \dot{\beta}}}{r}\right)=0 .
$$

From (2.54) and (2.59) it follows then

$$
\nabla k_{\alpha \dot{\beta}}=0 .
$$

Having two types of indices and being $\nabla$-constant we can look at $k_{\alpha \dot{\alpha}}$ as a metric that converts dotted indices into undotted ones and vise versa. Indeed, for any $A_{\dot{\alpha}}$ we can define

$$
A_{\alpha}:=k_{\alpha}{ }^{\dot{\beta}} A_{\dot{\beta}},
$$

\footnotetext{
${ }^{7}$ For the planar case the existence of $s p(2)$ flat connection was independently confirmed by M.A. Vasiliev.
} 
which entails from $(2.54)$

$$
A_{\dot{\alpha}}=k_{\dot{\alpha}}^{\gamma} A_{\gamma} \text {. }
$$

One should be cautious with signs though as this rule implies

$$
A^{\dot{\alpha}} B_{\dot{\alpha}}=-A^{\alpha} B_{\alpha} .
$$

Components of (2.27) get unified under $k$-transformation in a sense that they transform into each other upon index conversion

$$
k_{\dot{\alpha}}^{\gamma} \varkappa_{\gamma \alpha}=v_{\alpha \dot{\alpha}}, \quad k_{\dot{\alpha}}^{\gamma} v_{\gamma \dot{\beta}}=\bar{\varkappa}_{\dot{\alpha} \dot{\beta}}, \quad k_{\alpha}^{\dot{\gamma}} v_{\beta \dot{\gamma}}=\varkappa_{\alpha \beta} .
$$

This suggests once one has metric $k_{\alpha \dot{\alpha}}$ the only independent brane structure is, say, $\varkappa_{\alpha \beta}$, while the rest result from it via index conversion. This fact is a mere consequence of the more general constraint (2.52).

Another observation is an analog of the vierbein postulate for $\nabla$. One can check out the following identity

$$
\nabla \mathbf{E}_{\alpha, \beta}=0
$$

where we have introduced the one-form

$$
\mathbf{E}_{\alpha, \beta}:=\frac{1}{r} k_{\beta} \dot{\gamma} \mathbf{e}_{\alpha \dot{\gamma}} .
$$

It will be convenient to decompose $\mathbf{E}_{\alpha, \beta}$ into its symmetric and tracefull part

$$
\mathbf{E}_{\alpha, \beta}=\mathbf{E}_{\alpha \beta}+\frac{1}{2} \epsilon_{\alpha \beta} \mathbf{E}, \quad \mathbf{E}_{\alpha \beta}=\mathbf{E}_{\beta \alpha} .
$$

Both components are therefore covariantly constant

$$
\nabla \mathbf{E}_{\alpha \beta}=\nabla \mathbf{E}=0
$$

In terms of these fields the new connection reduces to

$$
w_{\alpha \beta}=\omega_{\alpha \beta}-r \mathbf{E}_{\alpha \beta}, \quad \bar{w}_{\dot{\alpha} \dot{\beta}}=\bar{\omega}_{\dot{\alpha} \dot{\beta}}-r \mathbf{E}_{\dot{\alpha} \dot{\beta}} .
$$

In view of (2.59) $\frac{1}{r} K_{A B}$ is covariantly constant with respect to connection $\nabla$. The only Lorentz scalar $r$ that system (2.28)-(2.29) has in this case is not a constant as follows from $(2.43)$

$$
\mathrm{d} \frac{1}{r}=\mathbf{E}
$$

Properties (2.59) as well as (2.69) play an important role in solving HS equations within the black brane ansatz especially greatly facilitating analysis at non-linear level [38]. 


\section{$3 \quad$ Higher-spin equations}

In this section we consider free bosonic HS equations using the Vasiliev approach. The main reason for choosing this formalism is that it makes higher-order analysis readily accessible on one hand and quite user friendly in four dimensions due to spinorial language on the other. The price for that simplicity is an extra set of fields that one has to deal with on top of the Fronsdal ones. Speaking of exact solution this amounts to calculation of auxiliary fields starting from free level.

HS equations of motion naturally group into the sector of gauge fields and the sector of HS curvatures (Weyl tensors). The latter admits an analog of the Penrose transform that gives a tool for constructing solutions, while the former has a HS gauge freedom that one should make use of properly. In practice, the analysis of the gauge sector is most challenging.

In this approach fields are valued in higher-spin algebra, which in $d=4$ is given by the Weyl algebra spanned by all polynomials of the generating $Y_{A}=\left(y_{\alpha}, \bar{y}_{\dot{\alpha}}\right)$ modulo the relations

$$
\left[y_{\alpha}, y_{\beta}\right]_{*}=2 i \epsilon_{\alpha \beta}, \quad\left[y_{\alpha}, \bar{y}_{\dot{\beta}}\right]_{*}=0, \quad\left[\bar{y}_{\dot{\alpha}}, \bar{y}_{\dot{\beta}}\right]_{*}=2 i \epsilon_{\dot{\alpha} \dot{\beta}},
$$

where star-product $*$ can be chosen to be the Moyal one

$$
f(Y) * g(Y)=f(Y) e^{i \epsilon^{A B} \overleftarrow{\partial}_{A} \vec{\partial}_{B}} g(Y)
$$

In practice one uses the integral representation form for exponential formula (3.2)

$$
f * g=\frac{1}{(2 \pi)^{4}} \int \mathrm{d}^{2} u \mathrm{~d}^{2} \bar{u} \mathrm{~d}^{2} v \mathrm{~d}^{2} \bar{v} e^{i u_{\alpha} v^{\alpha}+i \bar{u}_{\dot{\alpha}} \bar{v}^{\dot{\alpha}}} f(y+u, \bar{y}+\bar{u}) g(y+v, \bar{y}+\bar{v}) .
$$

Vacuum of the theory satisfies the HS zero-curvature condition

$$
\mathrm{d} W+W * W=0,
$$

where $W=W(Y \mid x)$ is the one-form. The only polynomial solution of that equation different from zero is the AdS vacuum

$$
W_{0}=-\frac{i}{4}\left(\omega^{\alpha \beta} y_{\alpha} y_{\beta}+\bar{\omega}^{\dot{\alpha} \dot{\beta}} \bar{y}_{\dot{\alpha}} \bar{y}_{\dot{\beta}}+2 \mathbf{e}^{\alpha \dot{\beta}} y_{\alpha} \bar{y}_{\dot{\beta}}\right),
$$

which upon feeding in (3.4) results in Cartan structure equations for $\mathrm{AdS}_{4}$

$$
\begin{aligned}
\mathrm{d} \omega_{\alpha \beta}-\omega_{\alpha}{ }^{\gamma} \omega_{\gamma \beta}-\mathbf{e}_{\alpha}{ }^{\dot{\gamma}} \mathbf{e}_{\beta \dot{\gamma}} & =0, \\
\mathrm{~d} \mathbf{e}_{\alpha \dot{\alpha}}-\omega_{\alpha}{ }^{\beta} \mathbf{e}_{\beta \dot{\alpha}}-\bar{\omega}_{\dot{\alpha}}{ }^{\dot{\beta}} \mathbf{e}_{\alpha \dot{\beta}} & =0 .
\end{aligned}
$$

Any other $W$ satisfying (3.4) inevitably contains fields of any spin $s \geq 1$.

Gauge invariant sector describing HS Weyl tensors along with matter fields $(s=0$ and $\left.s=\frac{1}{2}\right)$ is encoded in the zero-form $C(Y \mid x)$ that satisfies the so-called twisted-adjoint covariant constancy condition

$$
\mathrm{d} C+W_{0} * C-C * \pi\left(W_{0}\right)=0,
$$


where $\pi\left(W_{0}\right)$ flips the sign of $y \bar{y}$ part in $W_{0}(3.5)$ and more generally

$$
\pi f(y, \bar{y})=f(-y, \bar{y})
$$

Self-dual part of HS Weyl tensors is stored in $C(y, 0)$, such that the spin $s \geq 0$ component is

$$
C_{\alpha_{1} \ldots \alpha_{2 s}}=\left.\frac{\partial}{\partial y^{\alpha_{1}}} \cdots \frac{\partial}{\partial y^{\alpha_{2 s}}} C(y, \bar{y})\right|_{\bar{y}=0} .
$$

Substituting (3.5) into (3.8) results in

$$
D C-i \mathbf{e}^{\alpha \dot{\alpha}}\left(y_{\alpha} \bar{y}_{\dot{\alpha}}-\frac{\partial}{\partial y^{\alpha}} \frac{\partial}{\partial \bar{y}^{\dot{\alpha}}}\right) C=0,
$$

where $D$ is the Lorentz covariant derivative

$$
D=\mathrm{d}+\omega^{\alpha \beta} y_{\alpha} \frac{\partial}{\partial y^{\beta}}+\bar{\omega}^{\dot{\alpha} \dot{\beta}} \bar{y}_{\dot{\alpha}} \frac{\partial}{\partial \bar{y}^{\dot{\beta}}} .
$$

Gauge fields are generated by one-form $w(y, \bar{y} \mid x)$ that contains spin $s$ HS potential stored in $\omega_{\alpha_{1} \ldots \alpha_{s-1}, \dot{\alpha}_{1} \ldots \dot{\alpha}_{s-1}}$ component of $w$ or, equivalently, in the one singled out by

$$
y^{\alpha} \frac{\partial}{\partial y^{\alpha}} w=\bar{y}^{\dot{\alpha}} \frac{\partial}{\partial \bar{y}^{\dot{\alpha}}} w=(s-1) w,
$$

while other components are auxiliary. $w$ is sourced by the Weyl module $C$. Its equation of motion can be obtained from the Vasiliev system in the form ${ }^{8}$ [79]

$$
D_{0} w=\frac{i \eta}{4} \mathbf{e}^{\alpha \dot{\gamma}} \mathbf{e}^{\beta} \dot{\gamma} \frac{\partial^{2}}{\partial y^{\alpha} \partial y^{\beta}} C(y, 0 \mid x)+\frac{i \bar{\eta}}{4} \mathbf{e}^{\gamma \dot{\alpha}} \mathbf{e}_{\gamma}^{\dot{\beta}} \frac{\partial^{2}}{\partial \bar{y}^{\dot{\alpha}} \partial \bar{y}^{\dot{\beta}}} C(0, \bar{y} \mid x),
$$

where

$$
D_{0}=\mathrm{d}+\left[W_{0}, \bullet\right]_{*}=\mathrm{d}+\omega^{\alpha \beta} y_{\alpha} \frac{\partial}{\partial y^{\beta}}+\bar{\omega}^{\dot{\alpha} \dot{\beta}} \bar{y}_{\dot{\alpha}} \frac{\partial}{\partial \bar{y}^{\dot{\beta}}}+\mathbf{e}^{\alpha \dot{\beta}}\left(y_{\alpha} \frac{\partial}{\partial \bar{y}^{\dot{\beta}}}+\bar{y}_{\dot{\beta}} \frac{\partial}{\partial y^{\alpha}}\right)
$$

is the $\mathrm{AdS}_{4}$ covariant derivative (cf. (2.31)). Parameter $\eta$ is an arbitrary complex constant which unless $\eta=1$ or $\eta=i$ breaks parity of the theory. Since $D_{0}^{2}=0,(3.4)$ solutions to (3.14) are defined up to a gauge freedom

$$
w \sim w+D_{0} \epsilon
$$

A particular solution of (3.8) $w$ and (3.14) $C$ breaks local gauge symmetry down to the leftover global one, which is parameterized by $\epsilon_{0}(y, \bar{y} \mid x)$

$$
D_{0} \epsilon_{0}=0, \quad \epsilon_{0} * C-C * \pi\left(\epsilon_{0}\right)=0 .
$$

\footnotetext{
${ }^{8}$ Note the (anti)holomorphic dependence within $C$ on the right hand side of (3.14). This is the feature of ultra-locality that persists on any HS background (3.4) as well as at least at next interaction order.
} 


\subsection{Unfolded Penrose transform}

Let us now establish the AdS HS analog of the flat Penrose transform (2.48) introduced in $[53,68]$. Similar transformation has also been given in [80] and further elaborated in context of relation between adjoint and twisted-adjoint HS modules in [81]. Its various aspects in context of solution generating technique as well as in HS holography were detailed and studied in recent years [54-56, 58, 59, 69, 82-84].

To this end we define twistor ${ }^{9} T_{A}$ as an object that covariantizes (3.1) on AdS background

$$
D_{0} T_{A}=0, \quad\left[T_{A}, T_{B}\right]_{*}=2 i \epsilon_{A B},
$$

where $D_{0}$ is given by (3.15). Equation (3.18) can be solved as

$$
T_{A}=\Lambda_{A}^{B} Y_{B},
$$

where since $T_{A}$ preserves commutation relations, $\Lambda(x)$ should be an $\operatorname{Sp}(4)$ group element equal to identity at some locus point $x_{0}, \Lambda\left(x_{0}\right)=1$.

The Penrose transform is supposed to solve Weyl sector (3.8) of HS fields. In order to see this let us introduce distributions $\varkappa_{y}=2 \pi \delta^{2}(y)$ and $\bar{\varkappa}_{\bar{y}}=2 \pi \delta^{2}(\bar{y})$ which properties mimic (3.9). Using (3.3) one can show that

$$
F(y, \bar{y}) * \varkappa_{y}=\varkappa_{y} * F(-y, \bar{y}), \quad F(y, \bar{y}) * \bar{\varkappa}_{\bar{y}}=\bar{\varkappa}_{\bar{y}} * F(y,-\bar{y}),
$$

where $F(y, \bar{y})$ is an arbitrary function. Unlike standard distributions that can not be squared, the product of $\delta$-functions on non-abelian algebra (3.1) makes perfect sense

$$
\varkappa_{y} * \varkappa_{y}=\bar{\varkappa}_{\bar{y}} * \bar{\varkappa}_{\bar{y}}=1 \text {. }
$$

To reveal the meaning of the introduced distributions one can find using (3.3)

$$
F(y, \bar{y}) * \varkappa_{y}=\int \mathrm{d}^{2} u F(u, \bar{y}) e^{i u^{\alpha} y_{\alpha}},
$$

which shows that what $\varkappa_{y}$ does to a function is just the half Fourier transform. This trick allows us solving (3.8) in terms of an arbitrary twistor function with constant coefficients $F(T)$. Indeed, since from (3.18) it follows that

$$
D_{0} F(T)=\mathrm{d} F+W_{0} * F-F * W_{0}=0,
$$

and therefore to solve (3.8) one needs an extra twist which can be arranged using either $\varkappa_{y}$ or $\bar{\varkappa}_{\bar{y}}$. This gives us, in particular,

$$
C=F(T) * \varkappa_{y}
$$

as a solution of the twisted-adjoint flatness condition (3.8). Equation (3.24) and the similar conjugate one with $\bar{\varkappa}_{\bar{y}}$ in place of $\varkappa_{y}$ will be referred to as the unfolded Penrose transform. Explicitly, from (3.22) it follows

$$
C(y, \bar{y} \mid x)=\int \mathrm{d}^{2} u F\left(\Lambda_{\alpha}{ }^{\beta} u_{\beta}+\Lambda_{\alpha}{ }^{\dot{\beta}} \bar{y}_{\dot{\beta}}, \Lambda_{\dot{\alpha}}{ }^{\beta} u_{\beta}+\Lambda_{\dot{\alpha}}{ }^{\dot{\beta}} \bar{y}_{\dot{\beta}}\right) e^{i u^{\alpha} y_{\alpha}} .
$$

\footnotetext{
${ }^{9}$ Having $s p(4)$ form $\epsilon_{A B}$ we no longer distinguish between twistors and dual twistors.
} 
The holomorphic part of spin $s$ Weyl tensor can be extracted by setting $\bar{y}=0$ via

$$
C_{\alpha_{1} \ldots \alpha_{2 s}} \sim \int \mathrm{d}^{2} u u_{\alpha_{1}} \ldots u_{\alpha_{2 s}} F\left(\Lambda_{\alpha}{ }^{\beta} u_{\beta}, \Lambda_{\dot{\alpha}}{ }^{\beta} u_{\beta}\right) .
$$

Equation (3.26) is an AdS analog of the flat Penrose transform (2.48) and has much in common. There is however some difference. The flat one is written down in the Cartesian coordinates that allows one imposing explicit incidence relation (2.47) which enters the final result, while in AdS we stayed covariant implying that the $x$-dependence of twistor $T$ is implicit and stored in (3.19). Another difference is while integration in (2.48) is carried out along a closed contour, in (3.26) it goes across the two-dimensional plane. Formally, one can rewrite (3.26) in the form of a contour integral by means of Stokes' formula

$$
\int \mathrm{d}^{2} u \partial_{\alpha} g^{\alpha}(u)=\oint_{\Gamma} \mathrm{d} u^{\alpha} u_{\alpha} g(u) .
$$

We prefer to keep integration over a plane for a reason. While the contour presentation is convenient when dealing with functions that have poles (see e.g. [65]) which in addition allows one cropping a particular spin out by adjusting the pole degree, we stay with functions that are formally expandable in $Y$ 's in order to have control over star-product calculation. As a result the unfolded Penrose transform (3.24) gives us a solution for all spins at once rather than for a given one in particular. Nevertheless, we effectively find solutions for any fixed spin doing this way, as we make advantage of in what follows.

More importantly, as opposed to (2.48), its HS counterpart (3.24) restores not only spin $s$ Weyl tensors but the whole module $C$ that contains all on-shell derivatives of the former. It allows us reaching global symmetries of the resulting solution in a straightforward manner. Indeed, from (3.17), (3.24) it follows that the leftover symmetries are those that star commute with $F(T)$

$$
[\epsilon, F(T)]_{*}=0 .
$$

For example, for solutions generated by some constant rank-2 field $K_{0}^{A B}=K^{A B}\left(x_{0}\right)$ one picks

$$
F=F\left(K^{A B}\left(x_{0}\right) T_{A} T_{B}\right)=f\left(K^{A B}(x) Y_{A} Y_{B}\right),
$$

where upon substitution (3.19)

$$
K^{A B}(x)=\Lambda_{C}^{A} K^{C D}\left(x_{0}\right) \Lambda_{D}^{B}
$$

depends on $x$ via $\mathrm{Sp}(4)$ group adjoint action that spreads $K\left(x_{0}\right)$ across the AdS. Function (3.29) generates HS Weyl tensors via (3.26) which have a certain space-time global symmetry $\epsilon(Y \mid x)$ spanned by billinears in $Y$. To find this symmetry one can take $\epsilon=$ $\xi^{A B}\left(x_{0}\right) T_{A} T_{B}=\xi^{A B}(x) Y_{A} Y_{B}$, which manifestly satisfies $D_{0} \epsilon=0$ provided $\xi^{A B}\left(x_{0}\right)$ are some constants. Plugging it in (3.17) and using (3.28) results in

$$
\left[\xi^{A B}\left(x_{0}\right) T_{A} T_{B}, K^{C D}\left(x_{0}\right) T_{C} T_{D}\right]_{*}=0,
$$

which using (3.18) boils down to

$$
[\xi, K]_{A B}=0
$$


As expected, the global symmetry represented by parameter $\xi$ comes from the centralizer of $K$. In Particular, there are at least two Killing vectors associated with $\xi_{1 A B}=K_{A B}$ and $\xi_{2 A B}=K_{A B}^{-1}$ for generic $K$. This fact is in agreement with the presence of the KY tensor in this case which generates two Killing vectors [62, 77].

That global symmetries are easy to grasp within this approach is thanks to the fact that the unfolded Penrose transform reproduces the whole Weyl module $C(Y \mid x)$, where the symmetry action is naturally realized via (3.17). The standard Penrose transform (2.48) seemingly lacks direct access to global symmetries of the solution it generates. ${ }^{10}$ Let us also point out that black hole like fields generated with the aid of the unfolded Penrose transform in [53] fall into a double copy class of solutions on AdS. Their flat cousins were considered from double copy perspective in e.g. [85].

\section{Solutions}

In this section we construct free static solutions with planar symmetry for any spin $s \geq 0$. For $s=2$ the corresponding Weyl tensor is given by (2.32), provided brane condition (2.36) is imposed. Our strategy is to first generate Weyl module $C$ (3.8) and then solve (3.14) that it sources. Let us note that the Fronsdal fields result readily from the Kerr-Schild ansatz. Indeed, as was shown in [78] a spin $s \geq 0$ can be solved by

$$
\phi_{m_{1} \ldots m_{s}}=\frac{m_{s}}{U} l_{m_{1}} \ldots l_{m_{s}},
$$

where $m_{s}$ are arbitrary constants, while $l_{m}$ and $U$ are the Kerr-Schild vector and the scalar function that $K_{A B}$ generates via (2.44). While (4.1) looks neat and naturally generalizes black hole metric (2.9) still it is not most suitable for higher order analysis since the auxiliary fields that govern HS interaction have quite complicated form within the KerrSchild setup. For parity even model with $\eta=1$ we find different still simple form of the solution to (3.14).

\subsection{Twisted-adjoint sector}

To find planar solutions for $C$ we use the unfolded Penrose transform with twistor function (3.29). This has to be supplemented with brane condition (2.36) that guarantees the required global symmetry (2.7) in accordance with (3.32). Let us take

$$
F=s \exp \left(\frac{i s}{2} K_{A B} Y^{A} Y^{B}\right)
$$

for (3.29), where $s$ is an arbitrary constant and $K_{A B}$ is the $\mathrm{AdS}_{4}$ global symmetry parameter (2.27). Simple Gaussian integration in (3.24) and the use of (2.39)-(2.41) gives the following result

$$
\begin{aligned}
F * \varkappa_{y} & =s \int d^{2} u^{\prime} e^{\frac{i s}{2} \varkappa_{\alpha \beta} u^{\prime \alpha} u^{\prime \beta}} \exp \left(\frac{i}{2 s r^{2}} \varkappa_{\alpha \beta} y^{\alpha} y^{\beta}-\frac{i}{r^{2}} \varkappa_{\alpha}{ }^{\beta} v_{\beta \dot{\alpha}} y^{\alpha} \bar{y}^{\dot{\alpha}}\right) \sim \\
& \sim \frac{1}{r} \exp \left(\frac{i}{2 s r^{2}} \varkappa_{\alpha \beta} y^{\alpha} y^{\beta}-\frac{i}{r^{2}} \varkappa_{\alpha}{ }^{\beta} v_{\beta \dot{\alpha}} y^{\alpha} \bar{y}^{\dot{\alpha}}\right),
\end{aligned}
$$

\footnotetext{
${ }^{10}$ We are grateful to $\mathrm{C}$. White for the discussion on this point.
} 
where $r$ is given in (2.42) and $\sim$ means the equality up to an irrelevant at this stage complex phase factor attributed to the fact that the gaussian integration in (4.3) is carried out with the complex quadratic form and therefore should be completed via analytic continuation. Note the absence of billinears in $\bar{y}$ in (4.3) as they cancel out due to brane condition (2.36) being present otherwise. Similarly,

$$
F * \bar{\varkappa}_{\bar{y}} \sim \frac{1}{r} \exp \left(-\frac{1}{2 s r^{2}} \bar{\varkappa}_{\dot{\alpha} \dot{\beta}} \bar{y}^{\dot{\alpha}} \bar{y}^{\dot{\beta}}-\frac{i}{r^{2}} \varkappa_{\alpha}{ }^{\beta} v_{\beta \dot{\alpha}} y^{\alpha} \bar{y}^{\dot{\alpha}}\right) .
$$

Now, since $s$ is arbitrary one can integrate (4.3) and (4.4) over $s$ with any measure ${ }^{11} \rho(s)$ still producing solutions for $C$ which can be written down in the following form

$$
C(y, \bar{y} \mid x)=\frac{1}{r} f\left(\frac{1}{2 r^{2}} \varkappa_{\alpha \beta} y^{\alpha} y^{\beta}\right) e^{-\frac{i}{r^{2}} \varkappa_{\alpha}{ }^{\beta} v_{\beta \dot{\alpha}} y^{\alpha} \bar{y}^{\dot{\alpha}}}+\frac{1}{r} \bar{f}\left(\frac{1}{2 r^{2}} \bar{\varkappa}_{\dot{\alpha} \dot{\beta}} \bar{y}^{\dot{\alpha}} \bar{y}^{\dot{\beta}}\right) e^{-\frac{i}{r^{2}} \varkappa_{\alpha}{ }^{\beta} v_{\beta \dot{\alpha}} y^{\alpha} \bar{y}^{\dot{\alpha}}},
$$

where $f$ is an arbitrary (complex) analytic in $y$ function. Since $r$ is real (2.42), solution (4.5) clearly satisfies usual HS reality condition (see eq. (3.9) for the definition of $\pi$ )

$$
C^{\dagger}=\pi(C) \text {. }
$$

Let us stress that the procedure of obtaining (4.5) via Penrose transform (3.24) should be viewed as a guiding principle at best. We are not going to use function $F(Y)$ anywhere in what follows as it can well happen to be nonanalytic in $Y$ 's (see for example the analogous (2.51)). Indeed, it is $f(y)$ rather than $F(Y)$ should be analytic in order to correspond to HS fields. Moreover, $f(y)$ is polynomial for finite amount of spins. In this case however the corresponding $F(Y)$ is not analytic which makes it problematic to work with within the HS algebra. ${ }^{12}$ The fact that (4.5) satisfies (3.11) for any $f(y)$ can be checked directly without any reference to the Penrose transform.

The Weyl tensors (4.5) generates are of $D$-type

$$
\begin{aligned}
C_{\alpha_{1} \ldots \alpha_{2 s}} & =\frac{m_{s}}{r^{2 s+1}} \varkappa_{\left(\alpha_{1} \alpha_{2}\right.} \ldots \varkappa_{\left.\alpha_{2 s-1} \alpha_{2 s}\right)}, \\
\bar{C}_{\dot{\alpha}_{1} \ldots \dot{\alpha}_{2 s}} & =\frac{\bar{m}_{s}}{r^{2 s+1}} \bar{\varkappa}_{\left(\dot{\alpha}_{1} \dot{\alpha}_{2}\right.} \ldots \bar{\varkappa}_{\left.\dot{\alpha}_{2 s-1} \dot{\alpha}_{2 s}\right)},
\end{aligned}
$$

where the mass-like parameters $m_{s}$ are

$$
m_{s}=\left.\left(\frac{\partial}{\partial x}\right)^{s} f\left(\frac{x}{2}\right)\right|_{x=0} .
$$

Particularly, the scalar $s=0$ corresponds to $\Delta=1$ boundary behavior

$$
C_{\Delta=1}=\frac{m_{0}}{r} .
$$

\footnotetext{
${ }^{11}$ For example, a spin $m$ field can be extracted via residue $\oint \mathrm{d} s s^{2 m-1} F * \varkappa_{y}$ around zero. Note that the contour integration can not be interchanged with the star product one in this case.

${ }^{12}$ This is a general phenomenon immanent to the unfolded Penrose transform for it relates an infinite dimensional twisted-adjoint HS module to a finite-dimensional adjoint one. In practice it transforms polynomials into distributions and vice versa. An infinite tower of spins is necessary to have regular functions on both sides of the Penrose transform.
} 
While $m_{s>0}$ can be complex corresponding to both the magnetic- and electric- like HS parameters, we restrict ourselves to the case of real $m_{s}=\bar{m}_{s}$ which implies $f=\bar{f}$. For $s=2$ the respective Weyl tensors are of $\mathrm{AdS}_{4}$ black brane (2.1) of mass $M=m_{2}$. Note, that $f$ which reproduces a single given spin $s$ is just $f(x)=m x^{s}$. Being no more than polynomial, all information on the Weyl tensor on-shell derivatives is stored in the exponential of (4.5), which turns out to be the same for any spin thanks to the degenerate brane condition (2.36). This exponential has a remarkable projector property which becomes crucial at non-linear level [38].

Before going into the details let us first make use of the convention introduced in (2.61)(2.62). We note that what appears in the exponential of (4.5) is exactly (2.54). This leads us to define

$$
\bar{y}_{\alpha}:=k_{\alpha}{ }^{\dot{\beta}} \bar{y}_{\dot{\beta}} .
$$

Having this notation, solution (4.5) takes the form

$$
C=\frac{1}{r}(f(x)+f(\bar{x})) e^{i y_{\alpha} \bar{y}^{\alpha}}, \quad x=\frac{1}{2 r^{2}} \varkappa_{\alpha \beta} y^{\alpha} y^{\beta}, \quad \bar{x}=\frac{1}{2 r^{2}} \varkappa_{\alpha \beta} \bar{y}^{\alpha} \bar{y}^{\beta} .
$$

Now, one recognizes the Fock projector

$$
e^{i y_{\alpha} \bar{y}^{\alpha}} * e^{i y_{\alpha} \bar{y}^{\alpha}}=e^{i y_{\alpha} \bar{y}^{\alpha}}, \quad e^{i y_{\alpha} \bar{y}^{\alpha}} * \pi\left(e^{i y_{\alpha} \bar{y}^{\alpha}}\right)=\delta^{(2)}(0)
$$

which forms a Fock vacuum state for the following creation and annihilation operators

$$
a^{ \pm}=y \pm \bar{y}, \quad a^{-} * e^{i y_{\alpha} \bar{y}^{\alpha}}=e^{i y_{\alpha} \bar{y}^{\alpha}} * a^{+}=0 .
$$

In this respect let us note that projectors greatly facilitate non-linear HS analysis and for this reason are often introduced for solving the non-linear Vasiliev equations. In [53], where spherically symmetric solution was considered, the mass-like HS parameters for infinitely many HS fields were fine tuned to form a projector. This is in sharp contrast with our planar case, where the Fock projector shows up for each spin $s$ field right away.

Bearing in mind that Fock projector tends to self-reproduce at nonlinear level (see [73] where it was noted at quadratic level in the AdS/CFT context) we rewrite (3.11) so as to separate the projector dependence. To this end we decompose Lorentz covariant derivative (3.12) by separating part that contains $s p(2)$ flat connection (2.69) the oscillator representation of which reads

$$
\nabla=D+\mathbf{w}^{\alpha \beta} y_{\alpha} \frac{\partial}{\partial y^{\beta}}+\overline{\mathbf{w}}^{\dot{\alpha} \dot{\beta}} \bar{y}_{\dot{\alpha}} \frac{\partial}{\partial y^{\dot{\beta}}}=D-r \mathbf{E}^{\alpha \beta}\left(y_{\alpha} \frac{\partial}{\partial y^{\beta}}-\bar{y}_{\alpha} \frac{\partial}{\partial \bar{y}^{\beta}}\right),
$$

where we used

$$
\frac{\partial}{\partial \bar{y}^{\dot{\alpha}}}=-k^{\beta} \dot{\alpha} \frac{\partial}{\partial \bar{y}^{\beta}} .
$$

This way twisted-adjoint equation (3.11) reduces to

$$
\nabla C+i r \mathbf{E}^{\alpha \beta}(y+i \bar{\partial})_{\alpha}(\bar{y}-i \partial)_{\beta} C+\frac{i r}{2} \mathbf{E}\left(y_{\alpha} \bar{y}^{\alpha}+\partial_{\alpha} \bar{\partial}^{\alpha}\right) C=0 .
$$


We can now define a current module ${ }^{13} T(y, \bar{y})$ by separating the Fock projector

$$
C(y, \bar{y})=T(y, \bar{y}) e^{i y_{\alpha} \bar{y}^{\alpha}} .
$$

Using that $\nabla e^{i y_{\alpha} \bar{y}^{\alpha}}=0$ we find from (4.17)

$$
\nabla T+i r \mathbf{E}^{\alpha \beta} \bar{\partial}_{\alpha} \partial_{\beta} T-\frac{r}{2} \mathbf{E}\left(y^{\alpha} \partial_{\alpha}+\bar{y}^{\alpha} \bar{\partial}_{\alpha}-i \partial_{\alpha} \bar{\partial}^{\alpha}+2\right) T=0
$$

It is straightforward to check that $T=\frac{1}{r}(f(x)+f(\bar{x}))$ solves (4.19) for any function $f$. There is however another solution with planar symmetry which is not on the list (4.12). Indeed,

$$
T_{\Delta=2}=\frac{1}{r^{2}}\left(1+i y_{\alpha} \bar{y}^{\alpha}\right)
$$

corresponding to a scalar field with the alternative boundary condition $\Delta=2$ does satisfy (4.17). It was not captured by the unfolded Penrose transform (4.3) and we can add it to (4.12) if necessary for the nonlinear analysis

$$
C_{\Delta=2}=\frac{m_{0}^{\prime}}{r^{2}}
$$

\subsection{Adjoint sector}

Solutions to (3.14) is much harder to find. The unfolded Penrose transform does not act in this sector, while equation (3.14) is not homogeneous being sourced by primary components of the Weyl module $C$. Therefore, the solution procedure is a reconstruction of HS potentials in terms of curvatures. The result is gauge dependent as is seen from (3.16) and can be quite complicated even for plane wave- like solutions, [71, 72]. In addition, what one needs is not only Fronsdal components of $w(y, \bar{y})$, which form is quite simple in the Kerr-Schild representation (4.1), but the whole adjoint module that contains auxiliary fields related to the Fronsdal ones via derivatives. These auxiliary fields contribute to interactions within the unfolded approach. In principle one can start from the physical components given by (4.1) and restore all auxiliary fields one by one. While doable in principle the result turns out to be not particularly encouraging. Instead, inspired by flat connection (2.69) we provide an ansatz that works in HS A-model corresponding to $\eta=\bar{\eta}=1$ in (3.14) and brings us to the solution for the whole module $w$ in a simple form.

Our strategy is as follows. Since the only variable which is not constant with respect to connection $\nabla$ is $r$ (2.70) we can rewrite (3.14) in terms of $\nabla$ and then propose an ansatz for $w(y, \bar{y} \mid x)$ that depends on $\nabla$ - covariantly constant fields (2.59), (2.68) as well as on $r$ thus reducing the space-time partial differential equation to the ordinary one with respect to $r$.

Proceeding this way we first rewrite (3.14) in $\nabla$ - covariant way by expressing (3.15) as

$$
D_{0}=\nabla+r \mathbf{E}^{\alpha \beta}(y-\bar{y})_{\alpha}(\partial+\bar{\partial})_{\beta}-\frac{r}{2} \mathbf{E}\left(y^{\alpha} \bar{\partial}_{\alpha}+\bar{y}^{\alpha} \partial_{\alpha}\right) .
$$

\footnotetext{
${ }^{13}$ A very similar decomposition was used in [30] for HS bulk-boundary analysis, where the corresponding $T$ appears to describe $3 d$ boundary currents. We expect the analogy to be far reaching and hence borrow the terminology.
} 
This leads us to the following form ${ }^{14}$ of $(3.14)$

$$
\begin{aligned}
& \left(\nabla+r \mathbf{E}^{\alpha \beta}(y-\bar{y})_{\alpha}(\partial+\bar{\partial})_{\beta}-\frac{r}{2} \mathbf{E}\left(y^{\alpha} \bar{\partial}_{\alpha}+\bar{y}^{\alpha} \partial_{\alpha}\right)\right) w= \\
& =\frac{i r^{2}}{4} \mathbf{E}^{\alpha \gamma} \mathbf{E}_{\gamma}{ }^{\beta}\left(\bar{\eta} \bar{\partial}_{\alpha} \bar{\partial}_{\beta} C(0, \bar{y})-\eta \partial_{\alpha} \partial_{\beta} C(y, 0)\right)+\frac{i r^{2}}{4} \mathbf{E}^{\alpha \beta} \mathbf{E}\left(\eta \partial_{\alpha} \partial_{\beta} C(y, 0)+\bar{\eta} \bar{\partial}_{\alpha} \bar{\partial}_{\beta} C(0, \bar{y})\right) .
\end{aligned}
$$

Substituting (4.12) into (4.23) one arrives at

$$
\begin{aligned}
& \left(\nabla+r \mathbf{E}^{\alpha \beta}(y-\bar{y})_{\alpha}(\partial+\bar{\partial})_{\beta}-\frac{r}{2} \mathbf{E}\left(y^{\alpha} \bar{\partial}_{\alpha}+\bar{y}^{\alpha} \partial_{\alpha}\right)\right) w= \\
& =\frac{i r}{4} \mathbf{E}^{\alpha \gamma} \mathbf{E}_{\gamma}^{\beta}\left(\bar{\eta} \bar{\partial}_{\alpha} \bar{\partial}_{\beta} f(\bar{y})-\eta \partial_{\alpha} \partial_{\beta} f(y)\right)+\frac{i r}{4} \mathbf{E}^{\alpha \beta} \mathbf{E}\left(\eta \partial_{\alpha} \partial_{\beta} f(y)+\bar{\eta} \bar{\partial}_{\alpha} \bar{\partial}_{\beta} f(\bar{y})\right) .
\end{aligned}
$$

To solve this equation we propose the following ansatz

$$
w=\mathbf{E}^{\alpha \beta} \int_{0}^{1} \mathrm{~d} \tau \rho(\tau) \partial_{\alpha} \partial_{\beta} g(\tau y+(1-\tau) \bar{y}),
$$

where $\partial$ differentiates the argument of $g$,

$$
\partial_{\alpha} g(\xi):=\frac{\partial}{\partial \xi^{\alpha}} g(\xi)
$$

and $\rho(\tau)$ is an unspecified function. The idea behind (4.25) is that upon substitution into (4.24) the result may acquire a form of a total derivative with respect to $\tau$, such that at $\tau=1$ it leads to $\partial_{\alpha} \partial_{\beta} f(y)$, and to $\bar{\partial}_{\alpha} \bar{\partial}_{\beta} f(\bar{y})$ at $\tau=0$ correspondingly, thus matching the right hand side. The particular dependence on frame fields $\mathbf{E}_{\alpha \beta}$ and $\mathbf{E}$ is also a part of our ansatz motivated by the presence of second derivatives on the right hand side of (4.24).

Let us check if (4.25) works. When acting on $w$, the left hand side of (4.24) splits into two two-form sectors $\mathbf{E}^{\alpha \gamma} \mathbf{E}_{\gamma}{ }^{\beta}$ and $\mathbf{E}^{\alpha \beta} \mathbf{E}$. The first one that has contribution from the second term on the l.h.s. of (4.24) only reads

$$
\mathbf{E}^{\alpha \beta}(y-\bar{y})_{\alpha}\left(\frac{\partial}{\partial y}+\frac{\partial}{\partial \bar{y}}\right)_{\beta} \mathbf{E}^{\gamma \delta} \int_{0}^{1} \mathrm{~d} \tau \rho(\tau) \partial_{\gamma} \partial_{\delta} g=\mathbf{E}^{\alpha \beta} \mathbf{E}^{\gamma \delta}(y-\bar{y})_{\alpha} \int_{0}^{1} \mathrm{~d} \tau \rho(\tau) \partial_{\beta} \partial_{\gamma} \partial_{\delta} g .
$$

With the aid of the Schouten identities

$$
\mathbf{E}^{\alpha \beta} \mathbf{E}^{\gamma \delta}=\frac{1}{4}\left(\epsilon^{\alpha \gamma} \mathbf{H}^{\beta \delta}+\epsilon^{\beta \gamma} \mathbf{H}^{\alpha \delta}+\epsilon^{\alpha \delta} \mathbf{H}^{\beta \gamma}+\epsilon^{\beta \delta} \mathbf{H}^{\alpha \gamma}\right), \quad \mathbf{H}^{\alpha \beta}=-\mathbf{E}^{\alpha \gamma} \mathbf{E}_{\gamma}{ }^{\beta}
$$

(4.27) is further reduced to

$$
-\frac{1}{2} \mathbf{H}^{\beta \gamma}(y-\bar{y})^{\alpha} \partial_{\alpha} \int_{0}^{1} \mathrm{~d} \tau \rho(\tau) \partial_{\beta} \partial_{\gamma} g=-\frac{1}{2} \mathbf{H}^{\alpha \beta} \int_{0}^{1} \mathrm{~d} \tau \rho(\tau) \frac{\partial}{\partial \tau} \partial_{\alpha} \partial_{\beta} g,
$$

where in the last line we made use of the following identity

$$
(y-\bar{y})^{\alpha} \partial_{\alpha} g(\tau y+(1-\tau) \bar{y})=\frac{\partial}{\partial \tau} g(\tau y+(1-\tau) \bar{y}) .
$$

\footnotetext{
${ }^{14}$ By introducing $a^{ \pm}=y \pm \bar{y}$, equation (4.23) takes a form very similar to the one that arises in bulkboundary analysis of [30].
} 
We see that the result indeed acquires a form of a total derivative. Partial integration results in

$$
\int_{0}^{1} \mathrm{~d} \tau \rho(\tau) \frac{\partial}{\partial \tau} \partial_{\beta} \partial_{\beta} g=\rho(1) \partial_{\beta} \partial_{\beta} g(y)-\rho(0) \bar{\partial}_{\beta} \bar{\partial}_{\beta} g(\bar{y})-\int_{0}^{1} \mathrm{~d} \tau \rho^{\prime}(\tau) \partial_{\beta} \partial_{\beta} g .
$$

In order to match the right hand side of (4.24) one sets $g=f, \rho=-1$ and $\eta=\bar{\eta}=1$ thus having

$$
w=-\frac{i}{2} \mathbf{E}^{\alpha \beta} \int_{0}^{1} \mathrm{~d} \tau \partial_{\alpha} \partial_{\beta} f(\tau y+(1-\tau) \bar{y}) .
$$

Sector $\mathbf{E}^{\alpha \gamma} \mathbf{E}_{\gamma}{ }^{\beta}$ is perfectly satisfied by our ansatz, provided $\eta=\bar{\eta}$ which corresponds to the HS $A$-model case. On the other hand we have no freedom left within (4.25), which suggests that the remaining sector $\mathbf{E}^{\alpha \beta} \mathbf{E}$ is either satisfied by default or the ansatz does not go through.

To check out if (4.32) solves $\mathbf{E}^{\alpha \beta} \mathbf{E}$ sector is less trivial. In fact it does not for generic $f(\xi)$, but in the case of

$$
f(\xi)=f\left(\frac{1}{2 r^{2}} \varkappa_{\alpha \beta} \xi^{\alpha} \xi^{\beta}\right),
$$

which precisely corresponds to Weyl module (4.12), it does go through. Being somewhat technical we leave this verification for appendix, still noting here that a reason other than just a mere coincidence for why (4.32) satisfies another constraint from sector $\mathbf{E}^{\alpha \beta} \mathbf{E}$ is not entirely clear to us.

As a result, the adjoint module is solved by simple formula (4.32). It corresponds to the gauge where $y$ and $\bar{y}$ enter in a totally symmetric way. Indeed, from (4.32) and (4.33) one can obtain the following component form for $w$

$$
w_{\alpha(m), \beta(n)}=\frac{1}{r^{2+m+n}} T_{\alpha(m) \beta(n) \gamma \delta} \mathbf{E}^{\gamma \delta},
$$

where $T_{\alpha_{1} \ldots \alpha_{2 k}} \sim \varkappa_{\left(\alpha_{1} \alpha_{2}\right.} \ldots \varkappa_{\left.\alpha_{2 k-1} \alpha_{2 k}\right)}$ is totally symmetric. Hence, of gauge that results in such $w$ we can refer to as of the symmetric one.

Summarizing here our findings, the HS potentials that corresponds to Weyl module (4.12) have the following form

$$
w=-\frac{i}{2} \mathbf{E}^{\alpha \beta} \int_{0}^{1} \mathrm{~d} \tau \partial_{\alpha} \partial_{\beta} f\left(\frac{1}{2 r^{2}} \varkappa_{\gamma \delta}(\tau y+(1-\tau) \bar{y})^{\gamma}(\tau y+(1-\tau) \bar{y})^{\delta}\right),
$$

where we recall that here $\partial$ differentiates with respect to $\tau y+(1-\tau) \bar{y}$. To get back to original variables $(2.27)$ one substitutes

$$
\bar{y}_{\alpha}=-\frac{1}{r^{2}} \varkappa_{\alpha}{ }^{\beta} v_{\beta}^{\dot{\alpha}} \bar{y}_{\dot{\alpha}}, \quad \mathbf{E}_{\alpha \beta}=-\frac{1}{2 r^{3}}\left(\varkappa_{\alpha}^{\gamma} v_{\gamma}^{\dot{\gamma}} \mathbf{e}_{\beta \dot{\gamma}}+\varkappa_{\beta}^{\gamma} v_{\gamma}^{\dot{\gamma}} \mathbf{e}_{\alpha \dot{\gamma}}\right) .
$$

Let us stress that the form of the final result for $w$ is hard to anticipate without being guided by the auxiliary $s p(2)$ flat connection (2.53). It plays an important role in our derivation of $w$ and remains so at the interaction level considered in [38]. 


\section{Conclusion}

We have initiated a search for static solutions in $d=4$ higher-spin gauge theory that have planar symmetry. Owing to the standard gravity examples we expect such solutions may correspond to a thermal state of a boundary theory. The pursuit was encouraged by the solution generating technique of $[53,62]$ that produces black hole- like solutions from the AdS global symmetry parameter $K_{A B}$ for all spins at once. From the twistors standpoint this parameter is a rank-2 twistor and therefore can be fed into the Penrose transform yielding the Petrov $D$-type solutions. Since HS equations are based on the Vasiliev unfolded approach the Penrose transform gets modified accordingly and we call such a modification introduced in $[53,68]$ the unfolded Penrose transform. We provide details on that modification and compare it with the standard one. A major difference is the unfolded transform not only reproduces solutions to free HS Weyl tensors but as well to their all descendants which are on-shell derivatives thereof. This gives us, in particular, the access to the leftover global symmetries of the solutions it generates. In the planar case of our interest this implies the generating parameter should be degenerate satisfying (2.36).

Property (2.52) gives rise a certain $s p(2)$ flat connection that makes the properly rescaled Lorentz components of parameter $K_{A B}$ covariantly constant tensors with respect to this connection. That made us use the $s p(2)$ covariant formalism in our analysis. Using the unfolded Penrose transform we have found a class of solutions of the HS Weyl module parameterized by a single arbitrary analytic in $y$ 's function that encodes an infinite set of HS mass-like parameters. These include a free scalar with $\Delta=1$ boundary condition and $s \geq 1$ gauge fields that have the double copy origin. The function is of homogeneity degree $s$ for a single spin $s$ field. While the solution for $s=0$ with $\Delta=2$ boundary condition was not captured by our Penrose transform, it nevertheless naturally exists. For $s=2$ our solution corresponds to the $\mathrm{AdS}_{4}$ black brane.

Remarkable property of the obtained solution is that it contains one and the same Fock-type projector for any spin $s \geq 0$. Projectors are known to appear in the AdS/CFT context as bulk-to-boundary propagators $[22,31,75]$. It is conceivable that at the full non-linear level it may play a role of some thermal state. The nonlinear analysis gets especially interesting in the presence of projectors as they tend to survive in interactions and eventually decouple from HS equations [73]. This is especially relevant in context of (non)locality of HS interactions. Indeed, the decoupling of the projector leaves one with HS equations that contain no more than polynomial interactions for the states bounded in spin.

While the obtained solutions are by construction of $D$ Petrov type, it is also interesting to check whether they stay so in interaction. It would be also interesting to see whether the pure $s=2$ field corresponding to AdS black brane at leading order induces higher spins in interaction. In order to be able to answer these kind of questions one has to have complete solution at free level that includes both the sector of gauge invariant curvatures and the one of gauge fields. While the former comes about from the Penrose transform, the latter can be difficult to find. One of the main result of this paper is a simple formula for HS gauge fields (4.32) that we were able to derive in parity even HS theory $\eta=\bar{\eta}=1$. 
It makes a further quadratic analysis readily accessible [38]. It would be also interesting to know if there is as simple generalization of formula (4.32) available for parity broken theory.

A great deal of simplification in our derivation of HS gauge fields results from the emergent $s p(2)$ flat connection. On a practical side this implied that the space-time partial derivative equations of motion get reduced to the ordinary one along the radial variable $r$. Our result for the field components of HS connections $w_{\alpha(m), \dot{\beta}(n)}$ from $w(y, \bar{y})$ turns out to be totally symmetric with respect to permutations in both group of indices. Finally, let us note that the flat connection trick can be also useful in different context. In particular, the bulk-to-boundary propagator for gauge fields written in $[73,86]$ can be found using similar trick.

\section{Acknowledgments}

We are grateful to Chris White for useful discussions and to Mitya Ponomarev and M.A. Vasiliev for valuable comments on the draft of the paper. VD acknowledges participation of Oleg Shaynkman at the early stage of this work. Finally, we would like to thank the anonymous Referee for many valuable comments. The research was supported by the RFBR grant No. 20-02-00208.

\section{A Explicit form of the generating parameter}

The AdS global symmetry parameter that generates planar solutions have particular convenient form in Poincare coordinates $(2.8)$. Its $s o(3,1)$ tensor components originating from Killing vector $\frac{\partial}{\partial t}$ are given in $(2.26)$. The $s p(4, R)$ spinor realization is easy to obtain by introducing the $s o(3,2) \gamma$-matrices $\left\{\gamma_{I}, \gamma_{J}\right\}=2 \eta_{I J}$, which can be taken to be the following $\gamma_{I}=\left(\gamma_{a}, \gamma_{5}\right)$

$$
\begin{aligned}
& \gamma_{0}=\left(\begin{array}{cc}
-i \sigma^{2} & 0 \\
0 & i \sigma^{2}
\end{array}\right), \gamma_{1}=\left(\begin{array}{cc}
\sigma^{1} & 0 \\
0 & \sigma^{1}
\end{array}\right), \gamma_{2}=\left(\begin{array}{cc}
\sigma^{3} & 0 \\
0 & \sigma^{3}
\end{array}\right), \gamma_{3}=\left(\begin{array}{cc}
0 & i \sigma^{2} \\
-i \sigma^{2} & 0
\end{array}\right), \\
& \gamma_{5}=\gamma_{0} \gamma_{1} \gamma_{2} \gamma_{3}=\left(\begin{array}{cc}
0 & i \sigma^{2} \\
i \sigma^{2} & 0
\end{array}\right) .
\end{aligned}
$$

We can now define

$$
K_{A B}=\frac{1}{2} K_{I J}\left(\gamma^{I} \gamma^{J}\right)_{A B}
$$

Simple computation with $\gamma$-matrices and (2.26) gives us the following final result for $K$

$$
K=\frac{1}{z} \gamma_{0}\left(\gamma_{1}+\gamma_{5}\right) .
$$

Clearly, $K^{2}=0$ while the radial scalar defined in $(2.42)$ is

$$
r=\frac{1}{z} .
$$


The explicit $s p(4)$ form of the planar subalgebra (2.7) that commutes with $K$ is also easy to find

$$
\begin{aligned}
T & =K=\frac{1}{2} \gamma_{0}\left(\gamma_{1}+\gamma_{5}\right), \\
P_{1} & =\frac{1}{2} \gamma_{2}\left(\gamma_{1}+\gamma_{5}\right), \\
P_{2} & =\frac{1}{2} \gamma_{3}\left(\gamma_{1}+\gamma_{5}\right), \\
L & =\frac{1}{2} \gamma_{2} \gamma_{3}
\end{aligned}
$$

with the commutation relations

$$
\left[T, P_{1,2}\right]=[T, L]=0, \quad\left[P_{1}, P_{2}\right]=0, \quad\left[P_{1}, L\right]=P_{2}, \quad\left[P_{2}, L\right]=-P_{1}
$$

that fulfill $u(1) \oplus i s o(2)$ algebra.

\section{B Derivation of $s p(2)$ connection}

To proceed with the first order correction in the one-form sector we have used flat connection $w_{\alpha \beta}, \bar{w}_{\dot{\alpha} \dot{\beta}}$ defined by (2.53). It was claimed to be flat which is synonymous to

$$
\mathrm{d} w_{\alpha \beta}-w_{\alpha}^{\gamma} w_{\gamma \beta}=0
$$

To check this fact one should use defining relations for $\mathrm{AdS}_{4}$ geometry (3.6), (3.7) and differential conditions on isometry parameters (2.28), (2.29). Differential of $k$ defined by (2.54) is given by

$$
\mathrm{d} k_{\alpha \dot{\alpha}}=2\left(\mathbf{e}_{\sigma \dot{\sigma}} k^{\sigma \dot{\sigma}}\right) k_{\alpha \dot{\alpha}}+\omega_{\alpha}^{\gamma} k_{\gamma \dot{\alpha}}+2 \mathbf{e}_{\alpha \dot{\alpha}}+\frac{1}{r^{2}} \mathbf{e}_{\beta}{ }^{\dot{\gamma}} v_{\alpha \dot{\gamma}} v_{\dot{\alpha}}^{\beta}+\bar{\omega}_{\dot{\alpha}}{ }^{\dot{\gamma}} k_{\alpha \dot{\gamma}}-\frac{1}{r^{2}} \varkappa_{\alpha}{ }^{\beta} \mathbf{e}_{\beta} \dot{\gamma} \bar{\varkappa}_{\dot{\alpha} \dot{\gamma}}
$$

Now one is in a position to compute differential of $w_{\alpha \beta}$ and product $w_{\alpha}^{\gamma} w_{\gamma \beta}$. Straightforward computation by virtue of Fierz identity yields

$$
\begin{array}{r}
\mathrm{d} w_{\alpha \beta}=\omega_{\alpha}{ }^{\gamma} \omega_{\gamma \beta}-\frac{1}{2} \omega_{\alpha}{ }^{\sigma} \mathbf{e}_{\sigma \dot{\gamma}} k_{\beta} \dot{\gamma}-\frac{1}{2} \omega_{\beta}{ }^{\sigma} \mathbf{e}_{\sigma \dot{\gamma}} k_{\alpha \dot{\gamma}}-\frac{1}{2} \mathbf{e}_{\alpha}{ }^{\dot{\gamma}} \omega_{\beta}{ }^{\sigma} k_{\sigma \dot{\gamma}}-\frac{1}{2} \mathbf{e}_{\beta}{ }_{\beta}^{\dot{\gamma}} \omega_{\alpha}{ }^{\sigma} k_{\sigma \dot{\gamma}}- \\
-\overline{\mathbf{H}}_{\dot{\gamma} \dot{\sigma}} k_{\alpha}{ }^{\dot{ }} k_{\beta}{ }^{\dot{\gamma}}+\frac{1}{2} \mathbf{H}_{\alpha \beta}+\frac{1}{2 r^{2}} \overline{\mathbf{H}}_{\dot{\gamma} \dot{\sigma}} v_{\beta}{ }^{\dot{\sigma}} v_{\alpha}{ }^{\dot{\gamma}}-\frac{1}{2 r^{2}} \overline{\mathbf{H}}_{\dot{\gamma} \dot{\sigma}} \varkappa_{\alpha \beta} \dot{\bar{\varkappa}} \dot{\gamma} \dot{\sigma}
\end{array}
$$

and

$$
\begin{array}{r}
w_{\alpha}^{\gamma} w_{\gamma \beta}=\omega_{\alpha}^{\gamma} \omega_{\gamma \beta}-\frac{1}{2} \omega_{\alpha}^{\gamma} \mathbf{e}_{\gamma \dot{\gamma}} k_{\beta}^{\dot{\gamma}}-\frac{1}{2} \omega_{\beta}^{\sigma} \mathbf{e}_{\sigma \dot{\gamma}} k_{\alpha}^{\dot{\gamma}}+\frac{1}{2} \omega_{\alpha}{ }^{\sigma} \mathbf{e}_{\beta} k_{\sigma \dot{\gamma}}-\frac{1}{2} \mathbf{e}_{\alpha}^{\dot{\gamma}} \omega_{\beta}^{\gamma} k_{\gamma \dot{\gamma}}+ \\
+\frac{1}{2} \mathbf{H}_{\alpha \beta}-\frac{1}{2} \overline{\mathbf{H}}_{\dot{\gamma} \dot{\sigma}} k_{\alpha}{ }^{\dot{\gamma}} k_{\beta}{ }^{\dot{\sigma}}
\end{array}
$$

Here $\mathbf{H}_{\alpha \beta}$ and $\overline{\mathbf{H}}_{\dot{\alpha} \dot{\beta}}$ are basic two-form defined as

$$
\mathbf{H}_{\alpha \beta}=\mathbf{e}_{\alpha \dot{\gamma}} \mathbf{e}_{\beta}^{\dot{\gamma}}, \overline{\mathbf{H}}_{\dot{\alpha} \dot{\beta}}=\mathbf{e}_{\gamma \dot{\alpha}} \mathbf{e}_{\dot{\beta}}^{\gamma}
$$


After several simple cancellations one finds

$$
\mathrm{d} w_{\alpha \beta}-w_{\alpha}^{\gamma} w_{\gamma \beta}=-\frac{1}{2} \overline{\mathbf{H}}_{\dot{\gamma} \dot{\sigma}} k_{\alpha}{ }^{\dot{\gamma}} k_{\beta}^{\dot{\sigma}}+\frac{1}{2 r^{2}} \overline{\mathbf{H}}_{\dot{\gamma} \dot{\sigma}} v_{\beta}^{\dot{\sigma}} v_{\alpha}^{\dot{\gamma}}-\frac{1}{2 r^{2}} \overline{\mathbf{H}}_{\dot{\gamma} \dot{\sigma}} \varkappa_{\alpha \beta} \bar{\varkappa}^{\dot{\gamma} \dot{\sigma}}
$$

Using definition of $k, r$ (given by (2.42)) and identity (2.40) one can show that

$$
\begin{aligned}
& k_{\alpha}{ }^{\dot{\gamma}} k_{\beta}{ }^{\dot{\sigma}}=\left(-\frac{1}{r^{2}} \varkappa_{\alpha}{ }^{\sigma} v_{\sigma} \dot{\gamma}\right)\left(-\frac{1}{r^{2}} \varkappa_{\beta}^{\xi} v_{\xi}^{\dot{\sigma}}\right)=-\frac{1}{r^{4}}\left(\varkappa_{\alpha}{ }^{\sigma} v_{\sigma}{ }^{\dot{\gamma}}\right)\left(v_{\beta} \dot{\xi}_{\bar{\varkappa}_{\dot{\xi}}}{ }^{\dot{\sigma}}\right)= \\
& =-\frac{1}{r^{4}}\left[\left(v_{\sigma}{ }^{\dot{\gamma}} v_{\beta}^{\dot{\xi}}-v_{\beta}{ }^{\dot{\gamma}} v_{\sigma} \dot{\xi}\right) \varkappa_{\alpha}{ }^{\sigma} \bar{\varkappa}_{\dot{\xi}}^{\dot{\sigma}}+v_{\beta}{ }^{\dot{\gamma}} v_{\sigma} \dot{\xi} \varkappa_{\alpha}{ }^{\sigma} \bar{\varkappa}_{\dot{\xi}}^{\dot{\sigma}}\right]=-\frac{1}{r^{4}}\left[r^{2} \epsilon_{\sigma \beta} \bar{\epsilon}^{\dot{\gamma} \dot{\xi}} \varkappa_{\alpha}{ }^{\sigma} \bar{\varkappa}_{\dot{\xi}}{ }^{\dot{\sigma}}+v_{\beta}{ }^{\dot{\gamma}} v_{\sigma} \dot{\xi} \varkappa_{\alpha}{ }^{\sigma} \bar{\varkappa}_{\dot{\xi}}{ }^{\dot{\sigma}}\right] .
\end{aligned}
$$

Previous identity allows one to bring (B.6) to the form

$$
d w_{\alpha \beta}-w_{\alpha}^{\gamma} w_{\gamma \beta}=\frac{1}{2 r^{4}} \overline{\mathbf{H}}_{\dot{\gamma} \dot{\sigma}} v_{\beta} \dot{\gamma} v_{\alpha} \dot{\xi} \varkappa_{\alpha}{ }_{\bar{\varkappa}_{\dot{\xi}}}^{\dot{\sigma}}+\frac{1}{2 r^{2}} \overline{\mathbf{H}}_{\dot{\gamma} \dot{\sigma}} v_{\beta}^{\dot{\sigma}} v_{\alpha} \dot{\gamma} .
$$

To simplify the remaining terms on the r.h.s. one should once again apply identity (2.40), hence one has

$$
\begin{aligned}
& \mathrm{d} w_{\alpha \beta}-w_{\alpha}^{\gamma} w_{\gamma \beta}=-\frac{1}{2 r^{4}} \overline{\mathbf{H}}_{\dot{\gamma} \dot{\sigma}} v_{\beta}{ }^{\dot{\gamma}} v_{\alpha}{ }^{\dot{\rho}} \bar{\varkappa}_{\dot{\rho}} \dot{\xi}_{\bar{\varkappa}_{\dot{\xi}}}^{\dot{\sigma}}+\frac{1}{2 r^{2}} \overline{\mathbf{H}}_{\dot{\gamma} \dot{\sigma}} v_{\beta}^{\dot{\sigma}} v_{\alpha}^{\dot{\gamma}}= \\
& =-\frac{1}{2 r^{4}} \overline{\mathbf{H}}_{\dot{\gamma} \dot{\sigma}} v_{\beta}{ }^{\dot{\gamma}} v_{\alpha}{ }^{\dot{\rho}} r^{2} \delta_{\dot{\rho}}^{\dot{\sigma}}+\frac{1}{2 r^{2}} \overline{\mathbf{H}}_{\dot{\gamma} \dot{\sigma}} v_{\beta}^{\dot{\sigma}} v_{\alpha} \dot{\gamma}=0 .
\end{aligned}
$$

Flatness of $\bar{w}_{\dot{\alpha} \dot{\beta}}$ can be checked analogously.

\section{Checking the adjoint module}

In solving (4.24) the two conditions should be satisfied separately. Indeed, being a differential two-form, (4.24) splits into the part proportional to $\mathbf{E}^{\alpha \gamma} \mathbf{E}_{\gamma}{ }^{\beta}$ and to $\mathbf{E}^{\alpha \beta} \mathbf{E}$. Each of these should be equal to zero. We have checked that (4.25) passes through $\mathbf{E}^{\alpha \gamma} \mathbf{E}_{\gamma}{ }^{\beta}$-part and fixes the form of solution (4.32) unambiguously. It is therefore win or lose for the remaining $\mathbf{E}^{\alpha \beta} \mathbf{E}$. Let us check here that (4.32) satisfies it too.

Sector $\mathbf{E}^{\alpha \beta} \mathbf{E}$ is contributed by the first and third terms of (4.24). Consider those in detail. The first term is

$$
\begin{aligned}
& \nabla\left(\mathbf{E}^{\beta \beta} \int_{0}^{1} \mathrm{~d} \tau \partial_{\beta} \partial_{\beta} f\left(\frac{1}{2 r^{2}} \varkappa_{\alpha \alpha}(\tau y+(1-\tau) \bar{y})^{\alpha(2)}\right)\right)= \\
& =-\mathbf{E}^{\beta \beta} \mathbf{E} \partial_{\beta} \partial_{\beta} \int_{0}^{1} \mathrm{~d} \tau \frac{f^{\prime}}{2 r} \varkappa_{\alpha \alpha}(\tau y+(1-\tau) \bar{y})^{\alpha(2)},
\end{aligned}
$$

where $f^{\prime}(x)=\frac{\mathrm{d}}{\mathrm{d} x} f(x)$. Let us now note that for $f(\tau y+(1-\tau) \bar{y})$ the differentiation over the argument can be rewritten as

$$
\partial f=\left(\frac{\partial}{\partial y}+\frac{\partial}{\partial \bar{y}}\right) f
$$

and thus (C.1) boils down to

$$
-\mathbf{E}^{\beta \beta} \mathbf{E}\left(\frac{\partial}{\partial y}+\frac{\partial}{\partial \bar{y}}\right)_{\beta(2)}^{2} \int_{0}^{1} \mathrm{~d} \tau \frac{f^{\prime}}{2 r} \varkappa_{\alpha \alpha}(\tau y+(1-\tau) \bar{y})^{\alpha(2)} .
$$


To calculate the third term in (4.24) we use that

$$
\left(y^{\alpha} \frac{\partial}{\partial \bar{y}^{\alpha}}+\bar{y}^{\alpha} \frac{\partial}{\partial y^{\alpha}}\right)\left(\frac{\partial}{\partial y}+\frac{\partial}{\partial \bar{y}}\right)_{\beta(2)}^{2}=\left(\frac{\partial}{\partial y}+\frac{\partial}{\partial \bar{y}}\right)_{\beta(2)}^{2}\left(y^{\alpha} \frac{\partial}{\partial \bar{y}^{\alpha}}+\bar{y}^{\alpha} \frac{\partial}{\partial y^{\alpha}}\right)-2\left(\frac{\partial}{\partial y}+\frac{\partial}{\partial \bar{y}}\right)_{\beta(2)}^{2} .
$$

Now, evaluating

$$
\left(y^{\alpha} \frac{\partial}{\partial \bar{y}^{\alpha}}+\bar{y}^{\alpha} \frac{\partial}{\partial y^{\alpha}}\right) f=\frac{f^{\prime}}{r^{2}} \varkappa_{\alpha \alpha}(\tau y+(1-\tau) \bar{y})^{\alpha}(\tau \bar{y}+(1-\tau) y)^{\alpha}
$$

and using that

$$
\frac{f^{\prime}}{r^{2}} \varkappa_{\alpha \alpha}(\tau y+(1-\tau) \bar{y})^{\alpha}(y-\bar{y})^{\alpha}=\frac{\partial}{\partial \tau} f
$$

we have

$$
\begin{aligned}
& {\left[\nabla-\frac{r}{2} \mathbf{E}\left(y^{\alpha} \frac{\partial}{\partial \bar{y}^{\alpha}}+\bar{y}^{\alpha} \frac{\partial}{\partial y^{\alpha}}\right)\right] \mathbf{E}^{\beta \beta} \int_{0}^{1} \mathrm{~d} \tau \partial_{\beta} \partial_{\beta} f(\tau y+(1-\tau) \bar{y})=} \\
& =\frac{r}{2} \mathbf{E}^{\beta \beta} \mathbf{E} \int_{0}^{1} \mathrm{~d} \tau(1-2 \tau) \frac{\partial}{\partial \tau} \partial_{\beta} \partial_{\beta} f(\tau y+(1-\tau) \bar{y})-r \mathbf{E}^{\beta \beta} \mathbf{E} \int_{0}^{1} \mathrm{~d} \tau \partial_{\beta} \partial_{\beta} f(\tau y+(1-\tau) \bar{y}) .
\end{aligned}
$$

Integrating by parts we finally obtain

$$
\begin{aligned}
& {\left[\nabla-\frac{r}{2} \mathbf{E}\left(y^{\alpha} \frac{\partial}{\partial \bar{y}^{\alpha}}+\bar{y}^{\alpha} \frac{\partial}{\partial y^{\alpha}}\right)\right] \mathbf{E}^{\beta \beta} \int_{0}^{1} \mathrm{~d} \tau \partial_{\beta} \partial_{\beta} f(\tau y+(1-\tau) \bar{y})=} \\
& =-\frac{r}{2} \mathbf{E}^{\beta \beta} \mathbf{E}\left(\partial_{\beta} \partial_{\beta} f(y)+\bar{\partial}_{\beta} \bar{\partial}_{\beta} f(\bar{y})\right)
\end{aligned}
$$

and so we conclude that (4.32) is the solution.

Open Access. This article is distributed under the terms of the Creative Commons Attribution License (CC-BY 4.0), which permits any use, distribution and reproduction in any medium, provided the original author(s) and source are credited.

\section{References}

[1] J.M. Maldacena, The Large $N$ limit of superconformal field theories and supergravity, Int. J. Theor. Phys. 38 (1999) 1113 [Adv. Theor. Math. Phys. 2 (1998) 231] [hep-th/9711200] [INSPIRE].

[2] S.S. Gubser, I.R. Klebanov and A.M. Polyakov, Gauge theory correlators from noncritical string theory, Phys. Lett. B 428 (1998) 105 [hep-th/9802109] [INSPIRE].

[3] E. Witten, Anti-de Sitter space and holography, Adv. Theor. Math. Phys. 2 (1998) 253 [hep-th/9802150] [InSPIRE].

[4] I.R. Klebanov and A.A. Tseytlin, Entropy of near extremal black p-branes, Nucl. Phys. B 475 (1996) 164 [hep-th/9604089] [INSPIRE].

[5] E. Witten, Anti-de Sitter space, thermal phase transition, and confinement in gauge theories, Adv. Theor. Math. Phys. 2 (1998) 505 [hep-th/9803131] [InSPIRE].

[6] I.R. Klebanov and A.M. Polyakov, AdS dual of the critical $O(N)$ vector model, Phys. Lett. B 550 (2002) 213 [hep-th/0210114] [INSPIRE]. 
[7] E. Sezgin and P. Sundell, Massless higher spins and holography, Nucl. Phys. B 644 (2002) 303 [Erratum ibid. 660 (2003) 403] [hep-th/0205131] [INSPIRE].

[8] R.G. Leigh and A.C. Petkou, Holography of the $N=1$ higher spin theory on AdS $S_{4}$, JHEP 06 (2003) 011 [hep-th/0304217] [inSPIRE].

[9] C. Fronsdal, Massless Fields with Integer Spin, Phys. Rev. D 18 (1978) 3624 [InSPIRE].

[10] A.K.H. Bengtsson, I. Bengtsson and L. Brink, Cubic Interaction Terms for Arbitrary Spin, Nucl. Phys. B 227 (1983) 31 [InSPIRE].

[11] E.S. Fradkin and M.A. Vasiliev, On the Gravitational Interaction of Massless Higher Spin Fields, Phys. Lett. B 189 (1987) 89 [INSPIRE].

[12] R.R. Metsaev, Generating function for cubic interaction vertices of higher spin fields in any dimension, Mod. Phys. Lett. A 8 (1993) 2413 [INSPIRE].

[13] M.A. Vasiliev, More on equations of motion for interacting massless fields of all spins in (3+1)-dimensions, Phys. Lett. B 285 (1992) 225 [InSPIRE].

[14] D. Sorokin, Introduction to the classical theory of higher spins, AIP Conf. Proc. 767 (2005) 172 [hep-th/0405069] [INSPIRE].

[15] M.A. Vasiliev, Higher spin gauge theories: Star product and AdS space, in The Many Faces of the Superworld. Yuri Golfand Memorial Volume, World Scientific (2000), pp. 533-610 [hep-th/9910096] [INSPIRE].

[16] X. Bekaert, S. Cnockaert, C. Iazeolla and M.A. Vasiliev, Nonlinear higher spin theories in various dimensions, in proceedings of the 1st Solvay Workshop on Higher Spin Gauge Theories, Brussels, Belgium, 12-14 May 2004, hep-th/0503128 [INSPIRE].

[17] V.E. Didenko and E.D. Skvortsov, Elements of Vasiliev theory, arXiv:1401.2975 [INSPIRE].

[18] N. Boulanger and P. Sundell, An action principle for Vasiliev's four-dimensional higher-spin gravity, J. Phys. A 44 (2011) 495402 [arXiv:1102.2219] [InSPIRE].

[19] C. Sleight and M. Taronna, Higher Spin Interactions from Conformal Field Theory: The Complete Cubic Couplings, Phys. Rev. Lett. 116 (2016) 181602 [arXiv:1603.00022] [INSPIRE].

[20] M.A. Vasiliev, From Coxeter Higher-Spin Theories to Strings and Tensor Models, JHEP 08 (2018) 051 [arXiv: 1804.06520] [INSPIRE].

[21] N. Misuna, On unfolded off-shell formulation for higher-spin theory, Phys. Lett. B 798 (2019) 134956 [arXiv:1905.06925] [INSPIRE].

[22] S. Giombi and X. Yin, Higher Spin Gauge Theory and Holography: The Three-Point Functions, JHEP 09 (2010) 115 [arXiv:0912.3462] [INSPIRE].

[23] J.M. Maldacena and A. Zhiboedov, Constraining Conformal Field Theories with A Higher Spin Symmetry, J. Phys. A 46 (2013) 214011 [arXiv:1112.1016] [inSPIRE].

[24] O. Aharony, G. Gur-Ari and R. Yacoby, $d=3$ Bosonic Vector Models Coupled to Chern-Simons Gauge Theories, JHEP 03 (2012) 037 [arXiv:1110.4382] [INSPIRE].

[25] S. Giombi, S. Minwalla, S. Prakash, S.P. Trivedi, S.R. Wadia and X. Yin, Chern-Simons Theory with Vector Fermion Matter, Eur. Phys. J. C 72 (2012) 2112 [arXiv:1110.4386] [INSPIRE]. 
[26] J.M. Maldacena and A. Zhiboedov, Constraining conformal field theories with a slightly broken higher spin symmetry, Class. Quant. Grav. 30 (2013) 104003 [arXiv:1204.3882] [INSPIRE].

[27] O. Aharony, S. Giombi, G. Gur-Ari, J.M. Maldacena and R. Yacoby, The Thermal Free Energy in Large N Chern-Simons-Matter Theories, JHEP 03 (2013) 121 [arXiv:1211.4843] [INSPIRE].

[28] S. Giombi and I.R. Klebanov, One Loop Tests of Higher Spin AdS/CFT, JHEP 12 (2013) 068 [arXiv: 1308.2337] [INSPIRE].

[29] M. Beccaria and A.A. Tseytlin, Higher spins in AdS 5 at one loop: vacuum energy, boundary conformal anomalies and AdS/CFT, JHEP 11 (2014) 114 [arXiv:1410.3273] [INSPIRE].

[30] M.A. Vasiliev, Holography, Unfolding and Higher-Spin Theory, J. Phys. A 46 (2013) 214013 [arXiv: 1203.5554] [INSPIRE].

[31] V.E. Didenko and E.D. Skvortsov, Exact higher-spin symmetry in CFT: all correlators in unbroken Vasiliev theory, JHEP 04 (2013) 158 [arXiv:1210.7963] [INSPIRE].

[32] O.A. Gelfond and M.A. Vasiliev, Operator algebra of free conformal currents via twistors, Nucl. Phys. B 876 (2013) 871 [arXiv:1301.3123] [INSPIRE].

[33] A. Campoleoni, S. Fredenhagen, S. Pfenninger and S. Theisen, Asymptotic symmetries of three-dimensional gravity coupled to higher-spin fields, JHEP 11 (2010) 007 [arXiv: 1008.4744] [INSPIRE].

[34] M. Henneaux and S.-J. Rey, Nonlinear $W_{\infty}$ as Asymptotic Symmetry of Three-Dimensional Higher Spin Anti-de Sitter Gravity, JHEP 12 (2010) 007 [arXiv: 1008.4579] [INSPIRE].

[35] M.R. Gaberdiel and R. Gopakumar, An AdS 3 Dual for Minimal Model CFTs, Phys. Rev. D 83 (2011) 066007 [arXiv: 1011.2986] [INSPIRE].

[36] M. Gutperle and P. Kraus, Higher Spin Black Holes, JHEP 05 (2011) 022 [arXiv: 1103.4304] [INSPIRE].

[37] S.H. Shenker and X. Yin, Vector Models in the Singlet Sector at Finite Temperature, arXiv:1109.3519 [INSPIRE].

[38] V.E. Didenko and A.V. Korybut, Planar solutions of higher-spin theory II: nonlinear corrections, in preparation.

[39] X. Bekaert, J. Erdmenger, D. Ponomarev and C. Sleight, Quartic AdS Interactions in Higher-Spin Gravity from Conformal Field Theory, JHEP 11 (2015) 149 [arXiv: 1508.04292] [INSPIRE].

[40] C. Sleight and M. Taronna, Higher-Spin Gauge Theories and Bulk Locality, Phys. Rev. Lett. 121 (2018) 171604 [arXiv: 1704.07859] [INSPIRE].

[41] A.C. Petkou, Evaluating the AdS dual of the critical $O(N)$ vector model, JHEP 03 (2003) 049 [hep-th/0302063] [INSPIRE].

[42] G. Barnich and M. Henneaux, Consistent couplings between fields with a gauge freedom and deformations of the master equation, Phys. Lett. B 311 (1993) 123 [hep-th/9304057] [INSPIRE].

[43] M.A. Vasiliev, Dynamics of Massless Higher Spins in the Second Order in Curvatures, Phys. Lett. B 238 (1990) 305 [INSPIRE]. 
[44] O.A. Gelfond and M.A. Vasiliev, Homotopy Operators and Locality Theorems in Higher-Spin Equations, Phys. Lett. B $\mathbf{7 8 6}$ (2018) 180 [arXiv:1805.11941] [InSPIRE].

[45] M.A. Vasiliev, Current Interactions and Holography from the 0-Form Sector of Nonlinear Higher-Spin Equations, JHEP 10 (2017) 111 [arXiv:1605.02662] [INSPIRE].

[46] V.E. Didenko, O.A. Gelfond, A.V. Korybut and M.A. Vasiliev, Homotopy Properties and Lower-Order Vertices in Higher-Spin Equations, J. Phys. A 51 (2018) 465202 [arXiv: 1807.00001] [INSPIRE].

[47] V.E. Didenko, O.A. Gelfond, A.V. Korybut and M.A. Vasiliev, Limiting Shifted Homotopy in Higher-Spin Theory and Spin-Locality, JHEP 12 (2019) 086 [arXiv:1909.04876] [INSPIRE].

[48] O.A. Gelfond and M.A. Vasiliev, Spin-Locality of Higher-Spin Theories and Star-Product Functional Classes, JHEP 03 (2020) 002 [arXiv: 1910.00487] [INSPIRE].

[49] V.E. Didenko, O.A. Gelfond, A.V. Korybut and M.A. Vasiliev, Spin-locality of $\eta^{2}$ and $\bar{\eta}^{2}$ quartic higher-spin vertices, JHEP 12 (2020) 184 [arXiv: 2009.02811] [INSPIRE].

[50] O.A. Gelfond and A.V. Korybut, Manifest form of the spin-local higher-spin vertex $\Upsilon_{\omega C C C}^{\eta \eta}$, Eur. Phys. J. C 81 (2021) 605 [arXiv:2101.01683] [InSPIRE].

[51] E. Sezgin and P. Sundell, An Exact solution of $4 D$ higher-spin gauge theory, Nucl. Phys. B 762 (2007) 1 [hep-th/0508158] [INSPIRE].

[52] C. Iazeolla, E. Sezgin and P. Sundell, Real forms of complex higher spin field equations and new exact solutions, Nucl. Phys. B 791 (2008) 231 [arXiv:0706.2983] [INSPIRE].

[53] V.E. Didenko and M.A. Vasiliev, Static BPS black hole in $4 d$ higher-spin gauge theory, Phys. Lett. B 682 (2009) 305 [Erratum ibid. 722 (2013) 389] [arXiv:0906.3898] [INSPIRE].

[54] C. Iazeolla and P. Sundell, Families of exact solutions to Vasiliev's 4D equations with spherical, cylindrical and biaxial symmetry, JHEP 12 (2011) 084 [arXiv:1107.1217] [INSPIRE].

[55] P. Sundell and Y. Yin, New classes of bi-axially symmetric solutions to four-dimensional Vasiliev higher spin gravity, JHEP 01 (2017) 043 [arXiv:1610.03449] [INSPIRE].

[56] C. Iazeolla and P. Sundell, 4D Higher Spin Black Holes with Nonlinear Scalar Fluctuations, JHEP 10 (2017) 130 [arXiv:1705.06713] [INSPIRE].

[57] C. Iazeolla and J. Raeymaekers, On big crunch solutions in Prokushkin-Vasiliev theory, JHEP 01 (2016) 177 [arXiv: 1510.08835] [INSPIRE].

[58] C. Iazeolla, E. Sezgin and P. Sundell, On Exact Solutions and Perturbative Schemes in Higher Spin Theory, Universe 4 (2018) 5 [arXiv: 1711.03550] [INSPIRE].

[59] R. Aros, C. Iazeolla, J. Noreña, E. Sezgin, P. Sundell and Y. Yin, FRW and domain walls in higher spin gravity, JHEP 03 (2018) 153 [arXiv:1712.02401] [INSPIRE].

[60] J. Bourdier and N. Drukker, On Classical Solutions of 4d Supersymmetric Higher Spin Theory, JHEP 04 (2015) 097 [arXiv: 1411.7037] [INSPIRE].

[61] A. David and Y. Neiman, Bulk interactions and boundary dual of higher-spin-charged particles, JHEP 03 (2021) 264 [arXiv: 2009. 02893] [INSPIRE].

[62] V.E. Didenko, A.S. Matveev and M.A. Vasiliev, Unfolded Dynamics and Parameter Flow of Generic $\mathrm{AdS}_{4}$ Black Hole, arXiv:0901.2172 [INSPIRE].

[63] V.E. Didenko, A.S. Matveev and M.A. Vasiliev, unpublished. 
[64] T. Adamo, Lectures on twistor theory, PoS Modave2017 (2018) 003 [arXiv:1712.02196] [INSPIRE].

[65] C.D. White, Twistorial Foundation for the Classical Double Copy, Phys. Rev. Lett. 126 (2021) 061602 [arXiv:2012.02479] [INSPIRE].

[66] M. Walker and R. Penrose, On quadratic first integrals of the geodesic equations for type \{22\} spacetimes, Commun. Math. Phys. 18 (1970) 265 [InSPIRE].

[67] R.M. Floyd, The dynamics of Kerr fields, Ph.D. Thesis, London University, London U.K. (1974).

[68] O.A. Gelfond and M.A. Vasiliev, $\mathrm{Sp}(8)$ invariant higher spin theory, twistors and geometric BRST formulation of unfolded field equations, JHEP 12 (2009) 021 [arXiv:0901.2176] [INSPIRE].

[69] Y. Neiman, The holographic dual of the Penrose transform, JHEP 01 (2018) 100 [arXiv: 1709.08050] [INSPIRE].

[70] V.E. Didenko, N.G. Misuna and M.A. Vasiliev, Charges in nonlinear higher-spin theory, JHEP 03 (2017) 164 [arXiv: 1512.07626] [INSPIRE].

[71] K.I. Bolotin and M.A. Vasiliev, Star product and massless free field dynamics in AdS 4 , Phys. Lett. B 479 (2000) 421 [hep-th/0001031] [INSPIRE].

[72] B. Nagaraj and D. Ponomarev, Spinor-helicity formalism for massless fields in AdS 4 . Part II. Potentials, JHEP 06 (2020) 068 [arXiv:1912.07494] [INSPIRE].

[73] V.E. Didenko and M.A. Vasiliev, Test of the local form of higher-spin equations via AdS/CFT, Phys. Lett. B 775 (2017) 352 [arXiv: 1705. 03440] [INSPIRE].

[74] A. David and Y. Neiman, Higher-spin symmetry vs. boundary locality, and a rehabilitation of dS/CFT, JHEP 10 (2020) 127 [arXiv:2006.15813] [INSPIRE].

[75] V.E. Didenko and E.D. Skvortsov, Towards higher-spin holography in ambient space of any dimension, J. Phys. A 46 (2013) 214010 [arXiv:1207.6786] [InSPIRE].

[76] V. Didenko, On higher-spin black brane, in proceedings of the 2nd Solvay Workshop on "Higher Spin Gauge Theories", Brussels, Belgium, 5-8 February 2013.

[77] Y. Yasui and T. Houri, Hidden Symmetry and Exact Solutions in Einstein Gravity, Prog. Theor. Phys. Suppl. 189 (2011) 126 [arXiv:1104.0852] [InSPIRE].

[78] V.E. Didenko, A.S. Matveev and M.A. Vasiliev, Unfolded Description of AdS 4 Kerr Black Hole, Phys. Lett. B 665 (2008) 284 [arXiv:0801.2213] [INSPIRE].

[79] M.A. Vasiliev, Consistent Equations for Interacting Massless Fields of All Spins in the First Order in Curvatures, Annals Phys. 190 (1989) 59 [INSPIRE].

[80] J. Engquist and P. Sundell, Brane partons and singleton strings, Nucl. Phys. B 752 (2006) 206 [hep-th/0508124] [INSPIRE].

[81] C. Iazeolla and P. Sundell, A Fiber Approach to Harmonic Analysis of Unfolded Higher-Spin Field Equations, JHEP 10 (2008) 022 [arXiv: 0806.1942] [INSPIRE].

[82] D. De Filippi, C. Iazeolla and P. Sundell, Fronsdal fields from gauge functions in Vasiliev's higher spin gravity, JHEP 10 (2019) 215 [arXiv:1905.06325] [INSPIRE].

[83] R. Aros, C. Iazeolla, P. Sundell and Y. Yin, Higher spin fluctuations on spinless $4 D$ BTZ black hole, JHEP 08 (2019) 171 [arXiv:1903.01399] [INSPIRE]. 
[84] C. Iazeolla, On boundary conditions and spacetime/fibre duality in Vasiliev's higher-spin gravity, PoS CORFU2019 (2020) 181 [arXiv: 2004.14903] [INSPIRE].

[85] R. Monteiro, D. O'Connell and C.D. White, Black holes and the double copy, JHEP 12 (2014) 056 [arXiv: 1410.0239] [INSPIRE].

[86] E. Sezgin, E.D. Skvortsov and Y. Zhu, Chern-Simons Matter Theories and Higher Spin Gravity, JHEP 07 (2017) 133 [arXiv:1705.03197] [INSPIRE]. 